\title{
Graded-Mesh Difference Schemes for Singularly Perturbed Two-Point Boundary Value Problems*
}

\author{
By Eugene C. Gartland, Jr.
}

\begin{abstract}
This paper is concerned with the numerical approximation by compact finitedifference schemes of differential operators of the form $L_{\varepsilon} u=\varepsilon u^{(m)}+\sum_{\nu=0}^{m-1} a_{\nu} u^{(\nu)}$ without turning points. The stability of $L_{\varepsilon}$ combined with various auxiliary conditions is discussed, and a representation result for solutions of problems involving it is proven. This representation decomposes the solution into a smooth outer component plus a decaying exponential layer term along the lines of the Method of Multiple Scales.

The stability of compact difference analogues of $L_{\varepsilon}$ is studied, and a stability result is proven which generalizes earlier work. This result encompasses, for example, discretizations of second-order problems that fail to possess a maximum principle. It allows for standard polynomial-based differences in outer regions (away from boundary layers) with uniform meshes, even though such schemes admit oscillatory solutions.

A family of finite-difference schemes based on an exponentially graded mesh and local polynomial basis functions is discussed. These schemes can be constructed to have arbitrarily high uniform order of convergence. To achieve a scheme of order $O\left(h^{K}\right)$, roughly $K$ times as many points are distributed inside the layer as outside. The high order is achieved by using extra local evaluations of the coefficient functions and source term of the problem. A rigorous discretization error analysis of these schemes, using the established stability and representation results, is given.
\end{abstract}

Numerical results exhibiting the performance of these schemes are presented and generalizations of the results in the paper are discussed.

1. Introduction. This paper is concerned with the numerical approximation by finite-difference methods of singularly perturbed two-point boundary value problems. It has its motivation in numerical evidence that suggests that one can (stably and accurately) use standard central differences to approximate the solution of model problems of the form $\varepsilon u^{\prime \prime}+u^{\prime}=0$ in outer regions where the mesh spacing is large compared to $\varepsilon$ provided that one does something better near the boundary layer. This is true despite the fact that such a discretization violates certain "reasonableness" conditions: it does not have a maximum principle and admits oscillatory solutions, contrary to the behavior of the underlying continuous problem. We are thus led to consider under what circumstances such discretizations can be stable and accurate and what tools can be used to analyze them.

Received April 24, 1986; revised December 1, 1987.

1980 Mathematics Subject Classification. Primary 65L10; Secondary 34E15.

Key words and phrases. Singular perturbations, boundary value problems, finite differences, stability, uniform methods, graded meshes.

* Research supported in part by NSF grant DMS-8602199. 
The analysis can be carried out for more general problems, and we therefore consider linear operators $L_{\varepsilon}$ and $B_{\varepsilon}$ of the form

$$
L_{\varepsilon} u:=\varepsilon u^{(m)}+\sum_{\nu=0}^{m-1} a_{\nu} u^{(\nu)}, \quad B_{\varepsilon} u:=\left(B_{\varepsilon, 1} u, \ldots, B_{\varepsilon, m} u\right),
$$

where $\varepsilon$ is positive and conditions on the coefficient functions $a_{\nu}$ and auxiliary linear functionals $B_{\varepsilon, \mu}$ are given below. We are interested in solving problems of the type

$$
L_{\varepsilon} u=f, \quad B_{\varepsilon} u=\gamma
$$

for prescribed $f$ in $L^{1}[0,1]$ and $\gamma$ in $R^{m}$. We wish for such problems to be "well posed" in a strong sense (uniformly in $\varepsilon$ ), which we now make precise.

Let $\||\cdot|\|_{\varepsilon}$ denote the weighted Sobolev norm

$$
\|u \mid\|_{\varepsilon}:=\max \left\{\|u\|_{\infty}, \ldots,\left\|u^{(m-2)}\right\|_{\infty}, \varepsilon\left\|u^{(m-1)}\right\|_{\infty}\right\} .
$$

We say that the pair $\left(L_{\varepsilon}, B_{\varepsilon}\right)$ is strongly uniformly stable if there exist constants $C$ and $\varepsilon_{0}$ such that

$$
\||u|\|_{\varepsilon} \leq C\left\{\left\|L_{\varepsilon} u\right\|_{1}+\left|B_{\varepsilon} u\right|\right\}, \quad 0<\varepsilon \leq \varepsilon_{0}
$$

for all sufficiently smooth functions $u$. Here $\|\cdot\|_{1}$ is the usual $L^{1}$-norm and $|\cdot|$ denotes the 1-norm of the $m$-vector $B_{\varepsilon} u$.

Conditions that are sufficient to guarantee strong uniform stability can be established by using the stability of the initial value problem. Let $I_{\varepsilon}$ denote the initial data operator

$$
I_{\varepsilon} u:=\left(u(0), \ldots, u^{(m-2)}(0), \varepsilon u^{(m-1)}(0)\right) .
$$

We have the following.

THEOREM 1.1. If the coefficients $a_{\nu}$ in (1.1) satisfy

(1) $a_{\nu} \in L^{1}[0,1], \nu=0, \ldots, m-1$, and

(2) $a_{m-1}(x) \geq \underline{a}>0$,

then the initial value problem associated with $L_{\varepsilon}$ is strongly uniformly stable in the sense that there exists a constant $C$ such that for all $\varepsilon>0$ and for all $u$ sufficiently smooth,

$$
\|u\| \|_{\varepsilon} \leq C\left\{\left\|L_{\varepsilon} u\right\|_{1}+\left|I_{\varepsilon} u\right|\right\} .
$$

Proof. Using the integrating factor $\exp \left(\frac{1}{\varepsilon} \int_{0}^{x} a_{m-1}\right)$ for the leading terms of $L_{\varepsilon} u$, we establish, for sufficiently smooth $u$,

$$
u^{(m-1)}(x)=u^{(m-1)}(0) e^{-\frac{1}{\varepsilon} \int_{0}^{x} a_{m-1}}+\varepsilon^{-1} \int_{0}^{x} e^{-\frac{1}{\varepsilon} \int_{t}^{x} a_{m-1}} b(t) d t
$$

where

$$
b:=L_{\varepsilon} u-\sum_{\nu=0}^{m-2} a_{\nu} u^{(\nu)}
$$

From this it follows that

$$
\varepsilon\left|u^{(m-1)}(x)\right| \leq \varepsilon\left|u^{(m-1)}(0)\right|+\int_{0}^{x}|b|
$$


and

$$
\int_{0}^{x}\left|u^{(m-1)}\right| \leq \underline{a}^{-1}\left\{\varepsilon\left|u^{(m-1)}(0)\right|+\int_{0}^{x}|b|\right\} .
$$

Now from Taylor's formula we know that

$$
\max _{\nu=0, \ldots, m-2}\left|u^{(\nu)}(x)\right| \leq(m-1) \cdot \max _{\nu=0, \ldots, m-2}\left|u^{(\nu)}(0)\right|+\int_{0}^{x}\left|u^{(m-1)}\right|,
$$

while

$$
\int_{0}^{x}|b| \leq \int_{0}^{x}\left|L_{\varepsilon} u\right|+\int_{0}^{x}\left\{\left(\sum_{\nu=0}^{m-2}\left|a_{\nu}\right|\right) \cdot \max _{\nu=0, \ldots, m-2}\left|u^{(\nu)}\right|\right\} .
$$

The result follows from these using Gronwall's inequality. The constant $C$ depends on $m, \underline{a}^{-1}$, and $\left\|a_{0}\right\|_{1}, \ldots,\left\|a_{m-2}\right\|_{1}$.

The above theorem is proved in [17] for the case of continuous coefficients. From the stability of the initial value problem, we can deduce the stability of the boundary value problem if the auxiliary linear functionals $\left\{B_{\varepsilon, \mu}\right\}$ are linearly independent on the null space of $L_{\varepsilon}, N\left(L_{\varepsilon}\right)$, in this case in a sense that is uniform in $\varepsilon$. The following can be proven along the lines of Theorem 9 in [17].

THEOREM 1.2. Assume that the coefficients $a_{\nu}$ satisfy the conditions of Theorem 1.1 and that the linear functionals $B_{\varepsilon, \mu}$ satisfy, for $0<\varepsilon \leq \varepsilon_{0}$, some $\varepsilon_{0}$,

(1) $\left|B_{\varepsilon, \mu} u\right| \leq C\left|\|u \mid\|_{\varepsilon}, \mu=1, \ldots, m\right.$, for some absolute constant $C$ and for all sufficiently smooth functions $u$, and

(2) $\left\{B_{\varepsilon, \mu}\right\}_{\mu=1}^{m}$ are uniformly linearly independent on $N\left(L_{\varepsilon}\right)$, in the sense that there exists a fundamental system $\left\{\phi_{\nu}\right\}_{\nu=1}^{m}$ for $L_{\varepsilon}$ that satisfies

$$
\left\|\mid \phi_{\nu}\right\|_{\varepsilon} \leq C, \quad 0<\varepsilon \leq \varepsilon_{0},
$$

and is such that the matrix $\left[B_{\varepsilon, \mu} \phi_{\nu}\right]$ is nonsingular and has an inverse, the norm of which can be bounded independently of $\varepsilon$ for $0<\varepsilon \leq \varepsilon_{0}$. Then $\left(L_{\varepsilon}, B_{\varepsilon}\right)$ is strongly uniformly stable in the sense of (1.3).

Notice that while it is customary to think in terms of boundary value problems, with conditions specified at the endpoints $x=0$ and $x=1$, the auxiliary conditions here can be more general than that and can include, in particular, multi-point conditions. Solutions of problems of the type (1.2) admit boundary layers near the endpoint $x=0$, and these must be taken into account in the development and analysis of approximation schemes. Asymptotic expansions for such solutions can be obtained by various techniques; the one that is most useful for our present purposes is the method of multiple scales (see, for example, [18] or [21]). We use it now to characterize solutions of the homogeneous equation $L_{\varepsilon} u=0$.

THEOREM 1.3. Granted sufficient smoothness of the coefficient functions $a_{\nu}$, the differential operator $L_{\varepsilon}$ admits, for all $\varepsilon$ sufficiently small, a fundamental system of the form $\left\{\phi_{0}, \ldots, \phi_{m-2}, \exp \left(-\frac{1}{\varepsilon} \int_{0}^{x} a_{m-1}\right) \psi\right\}$, where $\phi_{0}, \ldots, \phi_{m-2}$, and $\psi$ and their derivatives through any prescribed finite order can be bounded independently of $\varepsilon$.

Proof. We first construct "outer" functions $\phi_{\mu}, \mu=1, \ldots, m-2$, in the form of truncated perturbation expansions plus remainders. Fix a positive integer $K$. Seek

$$
\phi_{\mu}=\phi_{\mu, 0}+\varepsilon \phi_{\mu, 1}+\cdots+\varepsilon^{K} \phi_{\mu, K}+\varepsilon^{K+1} R_{\mu}
$$


The component functions are defined recursively according to

$$
L_{0} \phi_{\mu, 0}=0, \quad \phi_{\mu, 0}^{(\nu)}(0)=\delta_{\mu, \nu}, \quad \nu=0, \ldots, m-2
$$

and

$$
L_{0} \phi_{\mu, k}=-\phi_{\mu, k-1}^{(m)}, \quad \phi_{\mu, k}^{(\nu)}(0)=0, \quad \nu=0, \ldots, m-2, \quad k=1, \ldots, K .
$$

The remainder is then taken to satisfy

$$
L_{\varepsilon} R_{\mu}=-\phi_{\mu, K}^{(m)}, \quad R_{\mu}^{(\nu)}(0)=0, \quad \nu=0, \ldots, m-1 .
$$

Here $L_{0}$ is the reduced $((m-1)$-st order) operator corresponding to $\varepsilon=0$.

Now the functions $\phi_{\mu, k}$ are independent of $\varepsilon$ and will have bounded derivatives up to any order if the data are sufficiently smooth. The stability of the initial value problem associated with $L_{\varepsilon}$ (Theorem 1.1) guarantees us that

$$
R_{\mu}, R_{\mu}^{\prime}, \ldots, R_{\mu}^{(m-2)}=O(1), \quad R_{\mu}^{(m-1)}=O\left(\varepsilon^{-1}\right),
$$

while successive differentiations of the differential equation satisfied by $R_{\mu}$ give that

$$
R_{\mu}^{(m+\nu)}=O\left(\varepsilon^{-\nu-2}\right), \quad \nu=0,1, \ldots .
$$

It follows that $\varepsilon^{K+1} R_{\mu}$ (and hence $\phi_{\mu}$ ) has uniformly bounded derivatives through the order $m+K-1$.

The boundary layer function can be constructed in a similar way. Let $E(x)$ denote the exponential layer type function

$$
E(x):=e^{-\frac{1}{\varepsilon} \int_{0}^{x} a_{m-1}},
$$

and let $L_{\varepsilon}^{+}$denote the differential operator defined by

$$
L_{\varepsilon}\left[\varepsilon^{m-2} E(x) v\right]=E(x) L_{\varepsilon}^{+} v .
$$

This can be written in the form

$$
L_{\varepsilon}^{+} v=\varepsilon^{m-1} v^{(m)}+\varepsilon^{m-2} a_{m-1}^{+} v^{(m-1)}+\cdots+\varepsilon a_{2}^{+} v^{\prime \prime}+a_{1}^{+} v^{\prime}+a_{0}^{+} v,
$$

where the coefficients $a_{\nu}^{+}$are functions of the $a_{\nu}$ and their derivatives and

$$
a_{1}^{+}=(-1)^{m-1} a_{m-1}^{m-1}+O(\varepsilon) .
$$

It follows that $a_{1}^{+}(x) \neq 0$ for $\varepsilon$ sufficiently small. Moreover, it can be shown by simple estimates of the type used in the proof of Theorem 1.1, that $L_{\varepsilon}^{+}$satisfies a stability inequality of the form

$$
\begin{aligned}
& \|v\|_{\infty}, \varepsilon\left\|v^{\prime}\right\|_{\infty}, \ldots, \varepsilon^{m-1}\left\|v^{(m-1)}\right\|_{\infty} \\
& \quad \leq C\left\{\left\|L_{\varepsilon}^{+} v\right\|_{1}+|v(0)|+\varepsilon\left|v^{\prime}(0)\right|+\cdots+\varepsilon^{m-1}\left|v^{(m-1)}(0)\right|\right\} .
\end{aligned}
$$

We now seek the layer solution in the form

$$
\phi_{m-1}=\varepsilon^{m-2} E(x)\left\{\psi_{0}+\varepsilon \psi_{1}+\cdots+\varepsilon^{K} \psi_{K}+\varepsilon^{K+1} R\right\} .
$$

Substituting into $L_{\varepsilon} \phi_{m-1}=0$ and balancing like powers of $\varepsilon$ gives a sequence of problems for the $\psi_{k}$ functions of the form

$$
\begin{aligned}
a_{1}^{+} \psi_{0}^{\prime}+a_{0}^{+} \psi_{0} & =0, \quad \psi_{0}(0)=1, \\
a_{1}^{+} \psi_{k}^{\prime}+a_{0}^{+} \psi_{k} & =F_{k}\left(\psi_{0}, \ldots, \psi_{k-1}\right), \quad \psi_{k}(0)=0, \quad k=1, \ldots, K .
\end{aligned}
$$


Here $F_{k}$ is a complicated function of $\psi_{0}, \ldots, \psi_{k-1}$ and their derivatives up to a certain order. Note that since $a_{1}^{+}(x)$ does not vanish (for all $\varepsilon$ sufficiently small) the solutions of these problems are well behaved and $\psi_{0}(x)$ is also nonvanishing. The remainder can then be taken to satisfy

$$
L_{\varepsilon}^{+} R=F\left(\psi_{0}, \ldots, \psi_{K}\right), \quad R(0)=\cdots=R^{(m-1)}(0)=0 .
$$

The stability inequality above for $L_{\varepsilon}^{+}$plus successive differentiations of the equation satisfied by $R$ give

$$
R^{(k)}=O\left(\varepsilon^{-k}\right), \quad k=0,1, \ldots .
$$

So $\varepsilon^{m+K-1} R$ (and hence $\psi$ ) has uniformly bounded derivatives through order $m+$ $K-1$. Since $K$ was chosen arbitrarily, the only limitation is the smoothness of the original coefficient functions $a_{\nu}$.

The linear independence of these functions can be established directly by inspection of the Wronskian matrix $W\left[\phi_{0}, \ldots, \phi_{m-1}\right]$. In fact, the matrix

$$
\varepsilon \exp \left(\frac{1}{\varepsilon} \int_{0}^{x} a_{m-1}\right) W\left[\phi_{0}, \ldots, \phi_{m-1}\right]
$$

is equal to an $O(\varepsilon)$ perturbation of a matrix of the form

$$
\left[\begin{array}{cc}
W\left[\phi_{0,0}, \ldots, \phi_{m-2,0}\right] & * \\
0 & (-1)^{m-1} a_{m-1}^{m-1} \psi_{0}
\end{array}\right] .
$$

The determinant of this is nonzero because neither $\psi_{0}(x)$ nor $a_{m-1}(x)$ vanish and because the leading order functions $\phi_{0,0}, \ldots, \phi_{m-2,0}$ are constructed to be a fundamental system for the reduced differential operator.

This method of constructing asymptotic solutions is well known, and the asymptotic nature of the infinite series so generated has been established for the case of analytic data. It is also known in this case that these series can be differentiated term by term and maintain their asymptotic character. See the references in $[17$, Section 3.2], in particular [23]. The author was unable to find the precise information contained above concerning the bounds on the derivatives and remainders in the case of nonanalytic coefficients, and this is required in what follows.

Using this characterization of the null space of $L_{\varepsilon}$, we can prove a representation result for solutions of problems of the form (1.2). This result will be used (instead of the usual local Taylor expansions) to do the truncation error analysis for the schemes derived in Section 3.

THEOREM 1.4. The solution $u$ of (1.2) admits the representation

$$
u=v+\varepsilon^{m-2} w e^{-\frac{1}{\varepsilon} \int_{0}^{x} a_{m-1}},
$$

where $v$ and $w$ and their derivatives up to any prescribed finite order can be bounded independently of $\varepsilon$, granted sufficient smoothness of the data $a_{0}, \ldots, a_{m-1}$, and $f$.

Proof. An $O(1)$, smooth particular integral for $L_{\varepsilon} u=f$ can be constructed along the lines of the construction of the outer solutions in the proof of Theorem 1.3. The result then follows from Theorem 1.3 and the observation that if the layer function is to have a (uniformly) bounded $\||\cdot|\|_{\varepsilon}$-norm, as is implied by the assumed stability of $\left(L_{\varepsilon}, B_{\varepsilon}\right)$, then it must be scaled as above. 
This result generalizes a related result corresponding to the case $m=2$ proved in [9] (see also [8]). Problems of the type (1.2), especially in the case $m=2$, have seen a lot of attention for some time now. See, for example, the references in [9] for a list of some of the contributions. Much effort has been devoted to the construction of uniform-mesh schemes, and the author was able to show in [9] how one could construct such schemes of arbitrarily high order of convergence, uniform in $\varepsilon$. Here we concentrate on issues particular to mesh grading and its impact specifically on finite-difference methods. We will see that classical polynomial-based approaches are applicable in much greater generality than has been previously supposed; the main obstacle to stability seems to be a need to do a sufficient amount of "upwinding" when proceeding through the very narrow transition regions between "inner" and "outer" mesh spacings.

This work then bears a relationship, at least in terms of topics addressed, to work done in [4] and [24], for the case of finite-difference methods for scalar equations, to [1], [2], and [3], for the case of collocation methods for first-order vector systems, and to [14] for the case of difference methods for systems. We now take up the issue of discrete approximations to problems of the form (1.2).

2. Stability of Compact Difference Schemes. We consider discretizations of the differential operator (1.1) by finite differences that are compact (in the sense of Kreiss, cf. [13]), that is, difference operators that involve the minimum number of mesh points (in this case $m+1$ ) necessary to consistently approximate the highestorder derivative (in this case $m$ ). Give a (not necessarily uniform) mesh $0=x_{0}<$ $x_{1}<\cdots<x_{n}=1$, and define the notations

$$
h_{i}:=x_{i+1}-x_{i}, \quad h:=\max h_{i}, \quad h_{k}\left(x_{i}\right):=\frac{x_{i+k}-x_{i}}{k} .
$$

The difference operator associated with the subinterval $\left[x_{i}, x_{i+m}\right]$ will be of the general form

$$
L_{h, \varepsilon} u_{i}^{h}:=\alpha_{i, 0} u_{i}^{h}+\cdots+\alpha_{i, m} u_{i+m}^{h} .
$$

Such difference operators can be written in many different ways, and for our purposes in what follows, two alternate forms are convenient. In these matters we follow much of the notation and general ideas of [17]. Let $D^{\nu}$ denote the divideddifference operator

$$
D^{\nu} u_{i}^{h}:=\nu ! u^{h}\left[x_{i}, \ldots, x_{i+\nu}\right] .
$$

The difference operator $L_{h, \varepsilon}$ can be written

$$
L_{h, \varepsilon} u_{i}^{h}=\varepsilon D^{m} u_{i}^{h}+a_{m-1}^{h}\left(x_{i}\right)\left(\theta_{i} D^{m-1} u_{i}^{h}+\left(1-\theta_{i}\right) D^{m-1} u_{i+1}^{h}\right)+b_{h}\left(u^{h}\right)_{i}
$$

or

$$
L_{h, \varepsilon} u_{i}^{h}=\varepsilon r_{i} D^{m} u_{i}^{h}+a_{m-1}^{h}\left(x_{i}\right) D^{m-1} u_{i+1}^{h}+b_{h}\left(u^{h}\right)_{i}
$$

where

$$
\begin{aligned}
b_{h}\left(u^{h}\right)_{i}:= & a_{m-2,0}^{h}\left(x_{i}\right) D^{m-2} u_{i}^{h}+a_{m-2,1}^{h}\left(x_{i}\right) D^{m-2} u_{i+1}^{h} \\
& +a_{m-2,2}^{h}\left(x_{i}\right) D^{m-2} u_{i+2}^{h}+\sum_{\nu=0}^{m-3} a_{\nu}^{h}\left(x_{i}\right) D^{\nu} u_{i+1}^{h}
\end{aligned}
$$


Here the $a^{h}$ are some prescribed mesh functions. These last two representations break the operator up into the leading-order parts plus a noncompact discretization of the lower-order terms of the differential operator $L_{\varepsilon}$. The mesh function $\theta$ serves as an "upwind" / "downwind" weighting parameter. Many of our stability hypotheses will involve conditions on $\theta_{i}$. The coefficients in these last two expressions for $L_{h, \varepsilon}$ are not uniquely determined. However, we do always have the relationship

$$
r_{i}=1-\rho_{i} \theta_{i}, \quad \rho_{i}:=\frac{h_{m}\left(x_{i}\right) a_{m-1}^{h}\left(x_{i}\right)}{\varepsilon} .
$$

The local dimensionless parameter $\rho_{i}$ represents the ratio of the local mesh spacing to the singular-perturbation parameter $\varepsilon$. Analogous discrete auxiliary functionals

$$
B_{h, \varepsilon} u^{h}=\left(B_{h, \varepsilon, 1} u^{h}, \ldots, B_{h, \varepsilon, m} u^{h}\right)
$$

are assumed given; we will not go into the details of these.

We wish for our finite-difference operators to satisfy a strong uniform discrete stability property analogous to the one satisfied by the continuous operators (namely (1.3)). We define the following discrete norms:

$$
\begin{aligned}
\left\|D^{\nu} u^{h}\right\|_{h, \infty} & :=\max \left\{\left|D^{\nu} u_{i}^{h}\right|: i=0, \ldots, n-\nu\right\} ; \\
\left\|u^{h}\right\|_{h, \varepsilon} & :=\max \left\{\left\|u^{h}\right\|_{h, \infty}, \ldots,\left\|D^{m-2} u^{h}\right\|_{h, \infty}, \varepsilon\left\|D^{m-1} u^{h}\right\|_{h, \infty}\right\} \\
\left\|f^{h}\right\|_{h, 1} & :=\sum_{i=0}^{n-m} h_{m}\left(x_{i}\right)\left|f_{i}^{h}\right| .
\end{aligned}
$$

We say that the pair $\left(L_{h, \varepsilon}, B_{h, \varepsilon}\right)$ is strongly uniformly stable if there exist constants $C, \varepsilon_{0}$, and $h_{0}$ such that

$$
\left\|\mid u^{h}\right\|_{h, \varepsilon} \leq C\left\{\left\|L_{h, \varepsilon} u^{h}\right\|_{h, 1}+\left|B_{h, \varepsilon} u^{h}\right|\right\}, \quad 0<\varepsilon \leq \varepsilon_{0}, 0<h \leq h_{0}
$$

for all mesh functions $u^{h}$.

Now for nonsingularly perturbed difference operators, we have general stability results that tell us that if the original differential operator is stable and if the discrete operator is compact and consistent, then the discrete operator is guaranteed to be stable, for all $h$ sufficiently small-see, for example, [6], [11], or [13], or other references contained in [10]. Unfortunately, this is not the case here. It is easy to construct examples of compact difference schemes that are uniformly consistent but not uniformly stable. Consider, for instance, the simple differential equation $\varepsilon u^{\prime \prime}+u^{\prime}=0$ discretized using the Allen-Southwell scheme (which is exact for this operator) on $[0,1 / 2]$ while using downwind differences in the outer region $[1 / 2,1]$. This scheme is uniformly $O(h)$ consistent, but in the $\varepsilon \rightarrow 0$ limit, the discretization matrix is singular, having two identical rows.

It is therefore necessary to add something extra to obtain strong uniform stability for compact finite-difference schemes. Some necessary and sufficient conditions for coerciveness and ellipticity for related operators (on one Hilbert space to another) are established in [7] using Fourier transforms and principal symbols and the like. Fairly general stability results are presented in [17] subject to the following conditions on the difference coefficients:

$$
a_{m-1}^{h}\left(x_{i}\right) \geq \delta>0, \quad\left\|a_{\nu}^{h}\right\|_{h, \infty} \leq M, \quad 0 \leq \theta_{i} \leq \min \left\{1,1 / \rho_{i}\right\} .
$$


It is shown there that if the difference scheme is in the form (2.1) (additionally with $a_{m-2,0}^{h}=a_{m-2,2}^{h}=0$ ) and has coefficients that satisfy the conditions above, then uniform consistency implies strong uniform stability (for all $h$ sufficiently small).

Unfortunately, it is easy to construct stable schemes that fail to satisfy these sufficient conditions. In particular, any discretization of $\varepsilon u^{\prime \prime}+u^{\prime}=0$ that uses standard central differences in an outer region like $[1 / 2,1]$, say, will have $\theta_{i}=1 / 2$ there, which does not go to zero as $\varepsilon / h \rightarrow 0$, contrary to what the third condition above necessarily implies. We will now generalize the stability results of [17].

The key to obtaining our discrete strong stability result, just as in the continuous case, is the establishment of an a priori stability with respect to initial data. We make use of the identity

$$
D^{m-1} u_{i+1}^{h}=\frac{r_{i}}{s_{i}} D^{m-1} u_{i}^{h}+\frac{1}{\varepsilon} \cdot \frac{1}{s_{i}} h_{m}\left(x_{i}\right) g_{i}^{h},
$$

where $r_{i}=1-\rho_{i} \theta_{i}$ (as before) and

$$
s_{i}:=1+\rho_{i}\left(1-\theta_{i}\right), \quad g_{i}^{h}:=L_{h, \varepsilon} u_{i}^{h}-b_{h}\left(u^{h}\right)_{i} .
$$

From this there follows, for any integers $k \leq l$,

$$
D^{m-1} u_{l+1}^{h}=\frac{r_{k}}{s_{k}} \cdots \frac{r_{l}}{s_{l}} D^{m-1} u_{k}^{h}+\frac{1}{\varepsilon} \sum_{i=k}^{l} h_{m}\left(x_{i}\right) \frac{1}{s_{i}} \frac{r_{i+1}}{s_{i+1}} \cdots \frac{r_{l}}{s_{l}} g_{i}^{h} .
$$

Here and in the sequel we use the convention that

$$
\frac{r_{i+1}}{s_{i+1}} \cdots \frac{r_{l}}{s_{l}}=1, \quad \text { when } i=l \text {. }
$$

From these identities, one can appraise $\varepsilon\left|D^{m-1} u^{h}\right|$ and $\left|D^{m-2} u^{h}\right|$, using

$$
D^{m-2} u_{l+2}^{h}=D^{m-2} u_{k+1}^{h}+\sum_{i=k}^{l} h_{m-1}\left(x_{i+1}\right) D^{m-1} u_{i+1}^{h},
$$

and then the lower-order differences, using similar relationships. The main estimates that are needed are somewhat technical and involve establishing inequalities concerning certain sums of products of the ratios $r_{i} / s_{i}$. We collect these in the following lemma.

LEMMA 2.1. For mesh functions $r_{i}$ and $s_{i}$ defined as above and for $\nu=$ $1, \ldots, m$, the following inequalities are valid subject to the stated conditions on the parameter $\theta$ :

(1) $\sum_{i=k}^{l} h_{\nu}\left(x_{i+1}\right)\left|\frac{r_{k}}{s_{k}} \cdots \frac{r_{i}}{s_{i}}\right| \leq h_{\nu}\left(x_{k+1}\right), \quad \frac{1}{\rho_{i}}-\frac{h_{\nu}\left(x_{i+1}\right)}{h_{\nu}\left(x_{i+2}\right)} \leq \theta_{i} \leq 0$;

(2) $\sum_{i=k}^{l} h_{\nu}\left(x_{i+1}\right)\left|\frac{r_{k}}{s_{k}} \cdots \frac{r_{i}}{s_{i}}\right| \leq C \varepsilon, \quad 0 \leq \theta_{i} \leq \frac{1}{\rho_{i}}$

(3) $\left|\sum_{i=k}^{l} h_{\nu}\left(x_{i+1}\right) \frac{r_{k}}{s_{k}} \cdots \frac{r_{i}}{s_{i}}\right| \leq h_{\nu}\left(x_{k+1}\right)$,

$$
\frac{1}{\rho_{i}} \leq \theta_{i} \leq \min \left\{\frac{1}{\rho_{i}}+\frac{h_{\nu}\left(x_{i-1}\right)}{h_{\nu}\left(x_{i-1}\right)+h_{\nu}\left(x_{i}\right)}, \frac{1}{\rho_{i}}+\frac{1}{2}, 1\right\}
$$


Proof. The proofs of these three estimates all follow along the same lines; we will do the proof for part (1). We proceed by doing an induction argument in $k$. Fix $l$. For $k=l$, we have

$$
\sum_{i=l}^{l} h_{\nu}\left(x_{i+1}\right)\left|\frac{r_{l}}{s_{l}} \cdots \frac{r_{i}}{s_{i}}\right|=h_{\nu}\left(x_{l+1}\right) \frac{r_{l}}{s_{l}} \leq h_{\nu}\left(x_{l+1}\right)
$$

because $\left|r_{i} / s_{i}\right|=r_{i} / s_{i} \leq 1$ for $\theta_{i} \leq 0$. Assuming the validity of the inequality for a given $k$, we have

$$
\begin{aligned}
\sum_{i=k-1}^{l} h_{\nu}\left(x_{i+1}\right)\left|\frac{r_{k-1}}{s_{k-1}} \cdots \frac{r_{i}}{s_{i}}\right| & =\frac{r_{k-1}}{s_{k-1}}\left[h_{\nu}\left(x_{k}\right)+\sum_{i=k}^{l} h_{\nu}\left(x_{i+1}\right) \frac{r_{k}}{s_{k}} \cdots \frac{r_{i}}{s_{i}}\right] \\
& \leq \frac{r_{k-1}}{s_{k-1}}\left(h_{\nu}\left(x_{k}\right)+h_{\nu}\left(x_{k+1}\right)\right) .
\end{aligned}
$$

But

$$
\frac{1-\rho_{k-1} \theta_{k-1}}{1+\rho_{k-1}\left(1-\theta_{k-1}\right)}\left(h_{\nu}\left(x_{k}\right)+h_{\nu}\left(x_{k+1}\right)\right) \leq h_{\nu}\left(x_{k}\right)
$$

is equivalent to

$$
\frac{1}{\rho_{k-1}}-\frac{h_{\nu}\left(x_{k}\right)}{h_{\nu}\left(x_{k+1}\right)} \leq \theta_{k-1}
$$

the assumed lower bound on $\theta_{k-1}$, and the inequality is established. In part (2), it is convenient to first prove that the sum with $h_{\nu}\left(x_{i+1}\right)$ replaced by $h_{m}\left(x_{i+1}\right)$. $a_{m-1}^{h}\left(x_{i+1}\right)$ (which is equal to $\left.\varepsilon \rho_{i+1}\right)$ can be bounded by $\varepsilon$. The inequality in (2) can then be established with $C=m / \underline{a}$.

In part (3), essential use is made of the sign alternation of the successive terms in the sum, which is implied by the condition $1-\rho_{i} \theta_{i} \leq 0$. What is actually established (again by induction in $k$ ) is

$$
h_{\nu}\left(x_{k+1}\right) \frac{r_{k}}{s_{k}} \leq \sum_{i=k}^{l} h_{\nu}\left(x_{i+1}\right) \frac{r_{k}}{s_{k}} \cdots \frac{r_{i}}{s_{i}} \leq 0 .
$$

The hypotheses on $\theta_{i}$ are used in the following ways:

$$
\theta_{i} \leq 1 \Rightarrow s_{i}>0, \quad \frac{1}{\rho_{i}} \leq \theta_{i} \Rightarrow r_{i} \leq 0, \quad \theta_{i} \leq \frac{1}{\rho_{i}}+\frac{1}{2} \Rightarrow\left|\frac{r_{i}}{s_{i}}\right| \leq 1,
$$

and

$$
\theta_{i} \leq \frac{1}{\rho_{i}}+\frac{h_{\nu}\left(x_{i-1}\right)}{h_{\nu}\left(x_{i-1}\right)+h_{\nu}\left(x_{i}\right)} \Rightarrow h_{\nu}\left(x_{i-1}\right)+h_{\nu}\left(x_{i}\right) \frac{r_{i}}{s_{i}} \geq 0,
$$

which is needed in the induction step.

With these estimates established, we are now in a position to prove our initial value stability property. Let $I_{h, \varepsilon}$ denote the discrete initial data operator

$$
I_{h, \varepsilon} u^{h}:=\left(u_{0}^{h}, \ldots, D^{m-2} u_{0}^{h},\left(\varepsilon+h_{m}\left(x_{0}\right)\right) D^{m-1} u_{0}^{h}\right) .
$$

We have the following. 
THEOREM 2.2. Assume that the difference coefficients in (2.1) and (2.3) satisfy for some absolute constants $\delta$ and $C$ and for $i=0, \ldots, n-m$

(1) $a_{m-1}^{h}\left(x_{i}\right) \geq \delta>0$,

(2) $\left\|a_{m-2,0}^{h}\right\|_{h, \infty},\left\|a_{m-2,1}^{h}\right\|_{h, 1},\left\|a_{m-2,2}^{h}\right\|_{h, \infty} \leq C$,

(3) $\left\|a_{\nu}^{h}\right\|_{h, 1} \leq C, \quad \nu=0, \ldots, m-3$,

(4) $\min \left\{0, \frac{1}{\rho_{i}}-\frac{h_{m-1}\left(x_{i+1}\right)}{h_{m-1}\left(x_{i+2}\right)}\right\} \leq \theta_{i}$

$$
\leq \min \left\{\frac{1}{\rho_{i}}+\frac{h_{m-1}\left(x_{i-1}\right)}{h_{m-1}\left(x_{i-1}\right)+h_{m-1}\left(x_{i}\right)}, \frac{1}{\rho_{i}}+\frac{1}{2}, 1\right\} .
$$

Furthermore, assume that the number of changes that $\theta_{i}$ makes from $\theta_{i}<0$ to $0<\theta_{i}$ and from $\theta_{i}<1 / \rho_{i}$ to $1 / \rho_{i}<\theta_{i}$ remains bounded independent of $h$ and $\varepsilon$, and that the mesh is locally quasi-uniform (uniformly in $h$ and $\varepsilon$ ) at those interfaces. Then the discrete initial value problem associated with $L_{h, \varepsilon}$ is strongly uniformly stable in the sense that there exists a constant $C$ such that for all $\varepsilon>0$ and $0<h<1$,

$$
\left\|u^{h}\right\|_{h, \varepsilon} \leq C\left\{\left\|L_{h, \varepsilon} u^{h}\right\|_{h, 1}+\left|I_{h, \varepsilon} u^{h}\right|\right\}
$$

for all mesh functions $u^{h}$.

Proof. In what follows, we take $C$ to denote a generic constant that does not depend on $h$ or $\varepsilon$. We begin by establishing the validity of the inequality

$$
\begin{aligned}
\left|D^{m-2} u_{l+2}^{h}\right| & +\varepsilon\left|D^{m-1} u_{l+1}^{h}\right| \\
& \leq C\left\{\left|D^{m-2} u_{1}^{h}\right|+\varepsilon\left|D^{m-1} u_{0}^{h}\right|+\sum_{i=0}^{l} h_{m}\left(x_{i}\right)\left|g_{i}^{h}\right|\right\}
\end{aligned}
$$

for $l=0, \ldots, n-m$. First, it follows from (2.5) and the facts

$$
\theta_{i} \leq \frac{1}{\rho_{i}}+\frac{1}{2} \Rightarrow\left|\frac{r_{i}}{s_{i}}\right| \leq 1, \quad \theta_{i} \leq 1 \Rightarrow 0<\frac{1}{s_{i}} \leq 1
$$

that

$$
\varepsilon\left|D^{m-1} u_{l+1}^{h}\right| \leq \varepsilon\left|D^{m-1} u_{0}^{h}\right|+\sum_{i=0}^{l} h_{m}\left(x_{i}\right)\left|g_{i}^{h}\right| .
$$

Next, by assumption the mesh consists of a finite number (which does not grow with $n$ ) of intervals along which $\theta_{i}$ falls into one of the three ranges in Lemma 2.1. For any such interval $\left[x_{k}, x_{l}\right]$, we have (from that lemma)

$$
\left|\sum_{i=k}^{l} h_{m-1}\left(x_{i+1}\right) \frac{r_{k}}{s_{k}} \cdots \frac{r_{i}}{s_{i}}\right| \leq C\left(\varepsilon+h_{m-1}\left(x_{k+1}\right)\right) .
$$

Now again using (2.5) and a change of order of summation, we obtain

$$
\begin{aligned}
D^{m-2} u_{l+2}^{h}= & D^{m-2} u_{k+1}^{h}+\sum_{i=k}^{l} h_{m-1}\left(x_{i+1}\right) D^{m-1} u_{i+1}^{h} \\
= & D^{m-2} u_{k+1}^{h}+D^{m-1} u_{k}^{h} \cdot \sum_{i=k}^{l} h_{m-1}\left(x_{i+1}\right) \frac{r_{k}}{s_{k}} \cdots \frac{r_{i}}{s_{i}} \\
& +\frac{1}{\varepsilon} \sum_{i=k}^{l} h_{m}\left(x_{i}\right) g_{i}^{h} \frac{1}{s_{i}} \sum_{j=i}^{l} h_{m-1}\left(x_{j+1}\right) \frac{r_{i+1}}{s_{i+1}} \cdots \frac{r_{j}}{s_{j}}
\end{aligned}
$$


from which there follows

$$
\begin{aligned}
\left|D^{m-2} u_{l+2}^{h}\right| \leq & \left|D^{m-2} u_{k+1}^{h}\right|+\left|D^{m-1} u_{k}^{h}\right| \cdot C\left(\varepsilon+h_{m-1}\left(x_{k+1}\right)\right) \\
& +\frac{1}{\varepsilon} \sum_{i=k}^{l} h_{m}\left(x_{i}\right)\left|g_{i}^{h}\right| \frac{1}{s_{i}}\left[h_{m-1}\left(x_{i+1}\right)+C\left(\varepsilon+h_{m-1}\left(x_{i+2}\right)\right)\right] .
\end{aligned}
$$

In this we now utilize the estimates

$$
\begin{aligned}
h_{m-1}\left(x_{k+1}\right)\left|D^{m-1} u_{k}^{h}\right| & =h_{m-1}\left(x_{k+1}\right)\left|\frac{D^{m-2} u_{k+1}^{h}-D^{m-2} u_{k}^{h}}{h_{m-1}\left(x_{k}\right)}\right| \\
& \leq C\left(\left|D^{m-2} u_{k+1}^{h}\right|+\left|D^{m-2} u_{k}^{h}\right|\right)
\end{aligned}
$$

and

$$
\frac{1}{\varepsilon} \cdot \frac{1}{s_{i}} h_{m-1}\left(x_{i+1}\right) \leq C \frac{\rho_{i}}{1+\rho_{i}\left(1-\theta_{i}\right)} \leq 2 C,
$$

where this last inequality follows from the condition $\theta_{i} \leq 1 / \rho_{i}+1 / 2$, plus a similar bound on the term in the sum involving $h_{m-1}\left(x_{i+2}\right)$ to obtain

$$
\left|D^{m-2} u_{l+2}^{h}\right| \leq C\left\{\left|D^{m-2} u_{k+1}^{h}\right|+\left|D^{m-2} u_{k}^{h}\right|+\varepsilon\left|D^{m-1} u_{k}^{h}\right|+\sum_{i=k}^{l} h_{m}\left(x_{i}\right)\left|g_{i}^{h}\right|\right\} .
$$

The desired inequality, (2.6), follows by piecing together estimates like the above across the interfaces between the regions where $\theta_{i}$ changes from being less than zero to greater than zero or from being less than $1 / \rho_{i}$ to greater than $1 / \rho_{i}$, combined with (2.7).

From (2.6) we establish (using the definitions of $g^{h}$ and $b_{h}\left(u^{h}\right)$ )

$$
\begin{aligned}
& \left(1-h_{m}\left(x_{l}\right)\left|a_{m-2,2}^{h}\left(x_{l}\right)\right|\right)\left|D^{m-2} u_{l+2}^{h}\right|+\varepsilon\left|D^{m-1} u_{l+1}^{h}\right| \\
& \leq C\left\{\sum_{i=0}^{l} h_{m}\left(x_{i}\right)\left|L_{h, \varepsilon} u_{i}^{h}\right|\right. \\
& +\sum_{i=0}^{l} h_{m}\left(x_{i}\right)\left[\left|a_{m-2,0}^{h}\left(x_{i+1}\right)\right|+\left|a_{m-2,1}^{h}\left(x_{i}\right)\right|\right. \\
& \left.+\left|a_{m-2,2}^{h}\left(x_{i-1}\right)\right|+\sum_{\nu=0}^{m-3}\left|a_{\nu}^{h}\left(x_{i}\right)\right|\right] \\
& \max _{\nu=0, \ldots, m-2}\left|D^{\nu} u_{i+1}^{h}\right|+\left|D^{m-2} u_{1}^{h}\right| \\
& \left.+h_{m}\left(x_{0}\right)\left|a_{m-2,0}^{h}\left(x_{0}\right)\right|\left|D^{m-2} u_{0}^{h}\right|+\varepsilon\left|D^{m-1} u_{0}^{h}\right|\right\} .
\end{aligned}
$$

Using the relationships

$$
D^{\nu-1} u_{l+2}^{h}=D^{\nu-1} u_{1}^{h}+\sum_{i=0}^{l} h_{\nu}\left(x_{i+1}\right) D^{\nu} u_{i+1}^{h}
$$

for $\nu=1, \ldots, m-2$ together with the inequality above, we establish, for $h\left\|a_{m-2,2}^{h}\right\|_{h, \infty}<1$,

$$
\phi_{l+1}^{h} \leq C\left\{\sum_{i=0}^{l} h_{m}\left(x_{i}\right) A_{i}^{h} \phi_{i}^{h}+\sum_{i=0}^{l} h_{m}\left(x_{i}\right)\left|L_{h, \varepsilon} u_{i}^{h}\right|+\phi_{0}^{h}\right\}
$$


where

$$
\phi_{i}^{h}:=\max \left\{\left|u_{i+1}^{h}\right|, \ldots,\left|D^{m-2} u_{i+1}^{h}\right|, \varepsilon\left|D^{m-1} u_{i}^{h}\right|\right\}
$$

and

$$
A_{i}^{h}:=\left|a_{m-2,0}^{h}\left(x_{i+1}\right)\right|+\left|a_{m-2,1}^{h}\left(x_{i}\right)\right|+\left|a_{m-2,2}^{h}\left(x_{i-1}\right)\right|+\sum_{\nu=0}^{m-3}\left|a_{\nu}^{h}\left(x_{i}\right)\right| .
$$

It now follows from Gronwall's inequality that

$$
\left\|\phi^{h}\right\|_{h, \infty} \leq C \exp \left(\left\|A^{h}\right\|_{h, 1}\right)\left\{\left\|L_{h, \varepsilon} u^{h}\right\|_{h, 1}+\left|\phi_{0}^{h}\right|\right\}
$$

and finally

$$
\left\|u^{h}\right\|_{h, \varepsilon} \leq C\left\{\left\|L_{h, \varepsilon} u^{h}\right\|_{h, 1}+\left|I_{h, \varepsilon} u^{h}\right|\right\} .
$$

As a direct consequence of this theorem, we get the following, more easily applicable corollary.

COROLLARY 2.3. Suppose that the difference operator $L_{h, \varepsilon}$ can be written in the form

$$
\begin{aligned}
L_{h, \varepsilon} u_{i}^{h}= & \varepsilon D^{m} u_{i}^{h}+a_{m-1}^{h}\left(x_{i}\right)\left(\theta_{i} D^{m-1} u_{i}^{h}+\left(1-\theta_{i}\right) D^{m-1} u_{i+1}^{h}\right) \\
& +\sum_{\nu=0}^{m-2} a_{\nu}^{h}\left(x_{i}\right) D^{\nu} u_{i+1}^{h},
\end{aligned}
$$

where the difference coefficients satisfy the following assumptions:

(1) $a_{m-1}^{h}\left(x_{i}\right) \geq \delta>0$,

(2) $\left\|a_{m-1}^{h}\right\|_{h, \infty} \leq C$,

(3) $\left\|a_{\nu}^{h}\right\|_{h, 1} \leq C, \nu=0, \ldots, m-2$, and

(4) $\theta$ satisfies the condition (4) of Theorem 2.2 to leading order,

that is, $\theta=\tilde{\theta}+\delta \theta$, where $\tilde{\theta}$ satisfies the condition and

$$
\left|\delta \theta_{i}\right| \leq C \min \left\{h_{m-1}\left(x_{i}\right), h_{m-1}\left(x_{i+1}\right)\right\}
$$

Then the discrete initial value problem associated with $L_{h, \varepsilon}$ is strongly uniformly stable in the sense of Theorem 2.2 .

Proof. The difference operator can be written in the form

$$
\begin{aligned}
L_{h, \varepsilon} u_{i}^{h}= & \varepsilon D^{m} u_{i}^{h}+a_{m-1}^{h}\left(x_{i}\right)\left(\tilde{\theta}_{i} D^{m-1} u_{i}^{h}+\left(1-\tilde{\theta}_{i}\right) D^{m-1} u_{i+1}^{h}\right) \\
& +a_{m-1}^{h}\left(x_{i}\right) \delta \theta_{i} D^{m-1} u_{i}^{h}-a_{m-1}^{h}\left(x_{i}\right) \delta \theta_{i} D^{m-1} u_{i+1}^{h} \\
& +\sum_{\nu=0}^{m-2} a_{\nu}^{h}\left(x_{i}\right) D^{\nu} u_{i+1}^{h},
\end{aligned}
$$

the second part of which is equal to

$$
\begin{aligned}
-\frac{a_{m-1}^{h}\left(x_{i}\right) \delta \theta_{i}}{h_{m-1}\left(x_{i}\right)} D^{m-2} u_{i}^{h} & -\frac{a_{m-1}^{h}\left(x_{i}\right) \delta \theta_{i}}{h_{m-1}\left(x_{i+1}\right)} D^{m-2} u_{i+2}^{h}+\sum_{\nu=0}^{m-3} a_{\nu}^{h}\left(x_{i}\right) D^{\nu} u_{i+1}^{h} \\
+ & {\left[\frac{a_{m-1}^{h}\left(x_{i}\right) \delta \theta_{i}}{h_{m-1}\left(x_{i}\right)}+a_{m-2}^{h}\left(x_{i}\right)+\frac{a_{m-1}^{h}\left(x_{i}\right) \delta \theta_{i}}{h_{m-1}\left(x_{i+1}\right)}\right] D^{m-2} u_{i+1}^{h} . }
\end{aligned}
$$

This will now satisfy the assumptions of Theorem 2.2 . 
The conditions of Theorem 2.2 and Corollary 2.3, especially the ones concerning $\theta_{i}$, are rather complicated. What they say in certain extreme ranges is easy to decipher. In outer regions, the situation is typically characterized by a uniform mesh and a large ratio of $h$ to $\varepsilon$, equivalently, $1 / \rho_{i} \approx 0$. In this case, condition (4) essentially becomes

$$
-1 \leq \theta_{i} \leq \frac{1}{2}
$$

While in the inner region (inside the boundary layer), we would have $1 / \rho_{i}$ large together with moderate local mesh ratios, which would combine to produce the condition

$$
0 \leq \theta_{i} \leq 1
$$

In transition regions, the "active" constraint will typically be

$$
\theta_{i} \leq \frac{1}{\rho_{i}}+\frac{1}{2}
$$

This condition can be somewhat restrictive, as we will see in some simple examples in the next section.

Theorem 2.2 and Corollary 2.3 generalize the results of [17] (in particular Theorem 3 of that paper) in substantive ways at the expense of certain mild restrictions on the mesh. The requirement that $\theta_{i}$ make a finite number of changes from one range of values to another is not much of a constraint for the applications we have in mind; typically, one of these ranges will apply throughout each of the interior, transition, and outer regions constructed in Section 3. Also, all of the meshes of that section are locally quasi-uniform; so the boundedness of local mesh ratios at any interfaces is assured. The reward for these constraints over the results of [17], which have no restrictions on the mesh, is a much broader allowable range for the weighting parameter $\theta$. In particular, it is not required that $\theta \rightarrow 0$ as $\varepsilon / h \rightarrow 0$, and all of the previously mentioned simple examples concerning standard central differences and the like are covered by our theorem.

Now for one-dimensional problems like ours, this a priori stability estimate is pretty much the main story, in the sense that the strong uniform stability of our boundary value problem is equivalent to this, subject to the uniform linear independence of the auxiliary functionals on the null space of $L_{h, \varepsilon}$. To be precise, we can use the approach of Theorem 11 of [17] to prove

THEOREM 2.4. Assume the following:

(1) the continuous pair $\left(L_{\varepsilon}, B_{\varepsilon}\right)$ is strongly uniformly stable in the sense of (1.3),

(2) the discrete difference operator $L_{h, \varepsilon}$ satisfies the assumptions of Theorem 2.2 ,

(3) the discrete auxiliary operator $B_{h, \varepsilon}$ satisfies

$$
\left|B_{h, \varepsilon} u^{h}\right| \leq C||\left|u^{h}\right| \|_{h, \varepsilon}
$$

for any mesh function $u^{h}$ and for some absolute constant $C$, and

(4) the discrete problem is uniformly consistent on $N\left(L_{\varepsilon}\right)$,

in the sense that

$$
\left\|\left(L_{h, \varepsilon}-L_{\varepsilon}\right) \phi_{\nu}\right\|_{h, 1},\left|\left(B_{h, \varepsilon}-B_{\varepsilon}\right) \phi_{\nu}\right| \rightarrow 0, \quad \text { as } h \rightarrow 0
$$


uniformly in $\varepsilon$ for $\phi_{\nu}$ in the fundamental system for $L_{\varepsilon}$ satisfying

$$
L_{\varepsilon} \phi_{\nu}=0, \quad B_{\varepsilon, \mu} \phi_{\nu}=\delta_{\mu, \nu}, \quad \mu, \nu=1, \ldots, m .
$$

Then the discretization pair $\left(L_{h, \varepsilon}, B_{h, \varepsilon}\right)$ is strongly uniformly stable in the sense of (2.4).

3. Discretizations and Graded Meshes. We consider the construction of polynomial-based, compact finite-difference discretizations of our problem on a particular graded mesh. Our goal is to lay out a procedure for the construction of schemes that satisfy the stability theorems of the previous section and which have an order of convergence that is uniform in $\varepsilon$ and can be made as high as desired.

An exponentially graded mesh and its properties. We assume that the differential operator $L_{\varepsilon}$ has been normalized so that $a_{m-1}(0)=1$. Fix a positive integer $K$, and let $h$ be the prescribed outer mesh spacing. Construct the graded mesh according to $x_{0}=0, h_{0}=\varepsilon h, x_{1}=x_{0}+h_{0}$, and

$$
\begin{aligned}
h_{i} & =\min \left\{h, \varepsilon h e^{\frac{1}{K \varepsilon} \int_{0}^{x_{i}} a_{m-1}}, e h_{i-1}\right\}, \\
x_{i+1} & =x_{i}+h_{i}, \quad i=1,2, \ldots .
\end{aligned}
$$

Except for a little adjusting that must be done at the endpoint, the construction is simple and straightforward; its suitability for the discretizations we have in mind will become apparent when we do our truncation error analysis later in this section. The integer $K$ will be related to the order of the scheme we wish to construct; it determines that roughly $K$ times as many mesh points go into the layer as outside of it. A similar mesh was proposed in [4]; it is equivalent to a one-dimensional version of mesh-grading schemes for finite-element approximations (see [20]).

We assume that we are in a range of parameters where $K \varepsilon<h$. We distinguish two points $x^{*}$ and $x^{\prime}$ defined by

$$
h e^{\frac{1}{K \varepsilon} \int_{0}^{x^{*}} a_{m-1}}=K, \quad \varepsilon e^{\frac{1}{K \varepsilon} \int_{0}^{x^{\prime}} a_{m-1}}=1 .
$$

It follows that $x^{*}<x^{\prime}<1$, for $\varepsilon$ sufficiently small, and

$$
x^{*} \approx K \varepsilon \ln \frac{K}{h}, \quad x^{\prime} \approx K \varepsilon \ln \frac{1}{\varepsilon} .
$$

Essentially, these points subdivide the interval $[0,1]$ into three regions: the inner region, $\left[0, x^{*}\right]$, where the mesh spacing is computed from the formula

$$
h_{i}=\varepsilon h e^{\frac{1}{K \varepsilon} \int_{0}^{x_{i}} a_{m-1}}
$$

the transition region, $\left[x^{*}, x^{\prime}\right]$, where $h_{i}=e h_{i-1}$, and the outer region, $\left[x^{\prime}, 1\right]$, where the spacing is uniform, $h_{i}=h$. These follow from the observations

$$
x_{i} \leq x^{*} \Rightarrow h e^{\frac{1}{K \varepsilon} \int_{0}^{x_{i}} a_{m-1}} \leq K
$$

and

$$
\frac{h_{i+1}}{h_{i}}=e^{\frac{1}{K \varepsilon} \int_{x_{i}}^{x_{i+1}} a_{m-1}} \approx e^{\frac{h_{i}}{K \varepsilon}} \leq e .
$$

The condition $h_{i} \leq e h_{i-1}$ assures local quasi-uniformity: here

$$
h_{i-1} \leq h_{i} \leq e h_{i-1} \text {. }
$$


These meshes are not globally quasi-uniform, since

$$
\frac{h_{\max }}{h_{\min }}=\frac{h}{\varepsilon h}=\frac{1}{\varepsilon} \text {. }
$$

In the absence of this mesh-ratio restriction, the final mesh ratio (at the interface $x=x^{\prime}$ ), which is the biggest mesh ratio, could get quite large and could cause difficulties for the stability. In the case $K \varepsilon \geq h$, there is no transition region; the mesh is graded according to the exponential formula until $x_{i+1} \geq x^{\prime}$, equivalently, $h_{i} \geq h$.

It is difficult to pin down exactly how many mesh points go into the various regions. Numerical evidence indicates that the number of points in the graded region, $\left[0, x^{\prime}\right]$, is roughly $K / h$ (i.e., $K$ times the number of outer mesh points) plus a very slowly growing function of $1 / h$ and $1 / \varepsilon$. It can be shown, by an induction argument, that one can place $K / h$ mesh points before getting to the point where $h_{i+1} / h_{i}$ is greater than $e$, which would put that point somewhere between $K \varepsilon$ and $x^{*}$.

An idea of Markowich and Ringhofer [16] can be used to obtain a crude bound on the number of points in the inner region.

PROPOSITION. The number of mesh points in the inner region is less than or equal to $\mathrm{Ke} / \underline{a} h$.

Proof. Let $N_{I}$ denote the number of mesh points in this region. Then we have

$$
\begin{aligned}
N_{I} & =\sum_{i: x_{i}<x^{*}} \frac{h_{i+1}}{h_{i}} \frac{h_{i}}{h_{i+1}} \leq \frac{e}{\varepsilon h} \sum_{i} h_{i} e^{-\frac{1}{K \varepsilon} \int_{0}^{x_{i+1}} a_{m-1}} \\
& \leq \frac{e}{\varepsilon h} \int_{0}^{x^{*}} e^{-\frac{a x}{K \varepsilon}} \leq \frac{K e}{\underline{a} h} .
\end{aligned}
$$

The transition region contains very few points, and this we can also verify.

PROPOSITION. The number of mesh points in the transition region is of the order $\ln \ln (h / K \varepsilon)$.

Proof. Suppose we start at the point $x^{*}$ with $x_{0}=x^{*}$. Then

$$
h_{0}=\varepsilon h e^{\frac{1}{K \varepsilon} \int_{0}^{x^{*}} a_{m-1}}=K \varepsilon,
$$

by the definition of $x^{*}$, and $h_{k}=K \varepsilon e^{k}$, for $k=1,2, \ldots$, and

$$
\begin{aligned}
x_{k+1} & =x_{0}+h_{0}+\cdots+h_{k} \\
& =x^{*}+K \varepsilon\left(1+e+\cdots+e^{k}\right) \\
& >x^{*}+K \varepsilon e^{k} .
\end{aligned}
$$

It follows that the point $x_{k+1}$ will be beyond $x^{\prime}(\approx K \varepsilon \ln (1 / \varepsilon))$, if $e^{k} \geq$ $\ln (h / K \varepsilon)$.

The restriction $h_{i+1} \leq e h_{i}$ does increase the width of the transition region a little bit. In a similar way it can be shown that it requires on the order of $\ln (h / K \varepsilon)$ mesh points to get from $x^{*}$ to the point where $h_{i} \geq h$, thus putting the interface between the graded and outer regions a small amount beyond $x^{\prime}$. 
High-order difference schemes. We construct finite-difference schemes using a general framework developed by Doedel [5] and Lynch and Rice [15] - see also [19]. Seek a discretization of the form

$$
L_{h, \varepsilon} u_{i}^{h}=\sum_{j=1}^{J} \beta_{i, j} f\left(\xi_{i, j}\right), \quad i=0, \ldots, n-m,
$$

where $L_{h, \varepsilon} u_{i}^{h}$ is a compact finite-difference expression supported on $\left[x_{i}, \ldots, x_{i+m}\right]$ with which we will usually work in the unique form of Corollary 2.3. The points $\xi_{i, j}$ are auxiliary evaluation points or HODIE points, in the terminology of [15]; they will typically fall in the subinterval $\left[x_{i}, x_{i+m}\right]$ and may or may not be mesh points. Actually these auxiliary points only need to be located within an $O\left(h_{m}\left(x_{i}\right)\right)$ distance of the stencil points.

The difference coefficients $a_{\nu}^{h}$ and $\theta$ and the weights $\beta_{i, j}$ are determined by the conditions that the scheme be exact, in the sense that

$$
L_{h, \varepsilon} \phi\left(x_{i}\right)=\sum_{j=1}^{J} \beta_{i, j} L_{\varepsilon} \phi\left(\xi_{i, j}\right)
$$

for all $\phi$ in some $(m+J)$-dimensional space of approximating functions subject to the normalization

$$
\sum_{j=1}^{J} \beta_{i, j}=1
$$

For example, in the case $m=2$, the one-point rule exact on $\left\{1, x, x^{2}\right\}$ for $x_{-1}=-h, x_{0}=0$, and $x_{1}=k$ is given by

$$
\begin{aligned}
L_{h, \varepsilon} u_{-1}^{h}= & \varepsilon D^{2} u_{-1}^{h}+a_{1}(\xi)\left(\theta D u_{-1}^{h}+(1-\theta) D u_{0}^{h}\right) \\
& +a_{0}(\xi)\left[\frac{\xi(\xi-k)}{h(h+k)} u_{-1}^{h}+\frac{(\xi+h)(k-\xi)}{h k} u_{0}^{h}+\frac{(h+\xi) \xi}{(h+k) k} u_{1}^{h}\right]=f(\xi),
\end{aligned}
$$

with

$$
\theta=\frac{-2 \xi+k}{h+k}
$$

This contains as a special case, when $\xi=0$, the standard central difference scheme

$$
\varepsilon D^{2} u_{-1}^{h}+a_{1}(0)\left[\frac{k}{h+k} D u_{-1}^{h}+\frac{h}{h+k} D u_{0}^{h}\right]+a_{0}(0) u_{0}^{h}=f(0) .
$$

It is interesting to examine these two simple schemes with respect to their stability. For the central difference scheme above, we have $\theta=k /(h+k)$, which always satisfies $0 \leq \theta \leq 1$. However, the difficult stability restriction from Theorem 2.2 is given here by

$$
\theta \leq \frac{1}{\rho}+\frac{1}{2} \Leftrightarrow k \leq h+\frac{4 \varepsilon}{a_{1}(0)}
$$

Since our graded mesh satisfies $h \leq k$, this says that in regions where $\varepsilon$ is small compared to the local mesh spacing, the spacing must be nearly uniform. This is necessary in order to get sufficient cancellation in the sums of the type (3) in Lemma 2.1. Indeed, numerical evidence bears this out: computations for model problems, using a graded mesh as constructed in this section (with $K=1$ ), and with the standard central difference scheme (3.3), give rise to stability constants 
that grow with $\varepsilon-$ slowly at first, on the order of $\ln (1 / \varepsilon)$, but eventually blowing up and destroying the computed results. Whereas experiments identical to the above except with an upwinded scheme in the narrow transition region prove to be uniformly stable. This suggests a degree of "tightness" or optimality in the hypotheses of Theorem 2.2 .

For the one-evaluation-point rule above, a sufficient condition for stability becomes

$$
\frac{k-h}{4}-\frac{\varepsilon}{a_{1}(\xi)} \leq \xi \leq \frac{k}{2} .
$$

This will be satisfied if the auxiliary evaluation point $\xi$ is situated appropriately in the upwind part of the mesh cell, namely $[0, k]$. The restriction $k / 4 \leq \xi \leq k / 2$, for example, is sufficient. In fact, the conspicuous choice would seem to be $\xi=k / 2$, in which case $\theta=0$ and the leading-order part of the difference operator becomes a purely upwind approximation.

In general, these formulas are too complicated to work out by hand, and one must compute them locally either symbolically or numerically. In [9], the author considered implementing this procedure, for the case $m=2$ with uniform meshes, using local approximating functions of the form

$$
\left\{1, x, \ldots, x^{K}, e^{-\frac{1}{\varepsilon} \int_{0}^{x} a_{m-1}}, x e^{-\frac{1}{\varepsilon} \int_{0}^{x} a_{m-1}}, \ldots, x^{L} e^{-\frac{1}{\varepsilon} \int_{0}^{x} a_{m-1}}\right\} .
$$

There it was shown that a uniform $O\left(h^{K}\right)$ discretization could be constructed with the choices $L=K-1$ and $J=2 K-1$. Here we consider discretizations based on $P_{m+J-1}$, the space of polynomials of degree at most $m+J-1$, which we will refer to as purely polynomial-based schemes, and those based on $P_{m+J-2}$ plus the single layer function $\exp \left(-\frac{1}{\varepsilon} \int_{0}^{x} a_{m-1}\right)$. These latter schemes we will refer to as augmented polynomial-based schemes. In order to know that this procedure is well posed, we must analyze the local linear-algebraic problem that results from the conditions of exactness on these spaces.

To leading order in $h$ and $\varepsilon$, it is sufficient to look at the operator $\varepsilon u^{(m)}+$ $a_{m-1}\left(x_{i}\right) u^{(m-1)}$; for simplicity, we will consider $\varepsilon u^{(m)}+u^{(m-1)}$, which can be accomplished by local scaling. It follows then, that for this simplified operator, the conditions of exactness on $\left\{1, x, \ldots, x^{m-1}\right\}$ imply

$$
a_{0}^{h}=\cdots=a_{m-2}^{h}=0, \quad a_{m-1}^{h}=1,
$$

so that

$$
L_{h, \varepsilon} u_{i}^{h}=\varepsilon D^{m} u_{i}^{h}+\theta_{i} D^{m-1} u_{i}^{h}+\left(1-\theta_{i}\right) D^{m-1} u_{i+1}^{h} .
$$

Consider first the purely polynomial-based schemes. The condition of exactness on $x^{m}$ implies that

$$
\theta_{i}=\frac{1}{h_{m}\left(x_{i}\right)} \sum_{j=1}^{J} \beta_{i, j}\left(\bar{x}_{i}-\xi_{i, j}\right)
$$

where

$$
\bar{x}_{i}:=\frac{x_{i+1}+\cdots+x_{i+m}}{m}
$$

the "Gauss point" for the reduced operator $u^{(m-1)}$ note, higher-order rules are possible in this general procedure by appropriate choices of evaluation points, see [15]. If the local mesh spacing is small compared to $\varepsilon$, then our scheme is well defined. In particular, we have the following. 
THEOREM 3.1. The finite-difference weights $\beta_{i, j}$ in the purely polynomial-based scheme are uniquely determined for all $h_{m}\left(x_{i}\right)$ sufficiently small provided the ratio $h_{m}\left(x_{i}\right) / \varepsilon$ is sufficiently small. In this case, the resulting parameter $\theta_{i}$ satisfies (to leading order)

$$
0 \leq \theta_{i} \leq 1
$$

which is sufficient to guarantee stability in this situation.

Proof. The system of linear equations to determine the weights results from the conditions of exactness for functions $\phi$ in the collection $\left\{x^{m+1}, \ldots, x^{m+J-1}\right\}$ subject to the restriction $\phi\left(x_{i}\right)=\cdots=\phi\left(x_{i+m}\right)=0$ plus the normalization condition $\sum_{j=1}^{J} \beta_{i, j}=1$. The nonsingularity of this system can be established for all $h_{m}\left(x_{i}\right)$ and $\rho_{i}\left(=h_{m}\left(x_{i}\right) / \varepsilon\right)$ sufficiently small along the lines of [15]. The conditions on $\theta_{i}$ can be seen as follows.

Let $\overline{\bar{x}}_{i}$ denote the full "mesh cell mean"

$$
\overline{\bar{x}}_{i}:=\frac{x_{i}+\cdots+x_{i+m}}{m+1} .
$$

Exactness on the function $\phi=\left(x-x_{i}\right) \cdots\left(x-x_{i+m}\right) /(m+1)$ ! implies

$$
0=\sum_{j=1}^{J} \beta_{i, j} L_{\varepsilon} \phi\left(\xi_{i, j}\right)=\sum_{j=1}^{J} \beta_{i, j}\left[\varepsilon\left(\xi_{i, j}-\overline{\bar{x}}_{i}\right)+O\left(h_{m}^{2}\left(x_{i}\right)\right)\right],
$$

from which it follows that

$$
\sum_{j=1}^{J} \beta_{i, j} \xi_{i, j}=\overline{\bar{x}}_{i}+O\left(\rho_{i} h_{m}\left(x_{i}\right)\right)
$$

Now we have from (3.4) and the above,

$$
0 \leq \theta_{i} \Leftrightarrow 0 \leq \bar{x}_{i}-\overline{\bar{x}}_{i}+O\left(\rho_{i} h_{m}\left(x_{i}\right)\right)
$$

which will be true for $\rho_{i} h_{m}\left(x_{i}\right)$ sufficiently small (by local quasi-uniformity of the mesh). And, after some simplification,

$$
\theta_{i} \leq 1 \Leftrightarrow O\left(\rho_{i} h_{m}\left(x_{i}\right)\right) \leq\left(x_{i+m}-x_{i}\right)+\cdots+\left(x_{i+m}-x_{i+m-1}\right),
$$

which is also true for sufficiently small $\rho_{i} h_{m}\left(x_{i}\right)$.

This essentially takes care of the inner region; because throughout most of that, we have $h_{i}$ of the order of $\varepsilon h$. So $\rho_{i}$ will be small if the outer (maximum) mesh spacing $h$ is small compared to 1 .

When the ratio $\rho_{i}$ is large, it is possible for our local system to be inconsistent; it is easy to see this by looking at the system corresponding to a two-point rule for $\varepsilon u^{\prime \prime}+u^{\prime}$ constructed to be exact on $\left\{1, x, x^{2}, x^{3}\right\}$. It is the case, however, that the under-determined system, exact on $P_{m+J-2}$ instead of $P_{m+J-1}$, is always consistent. This can be seen either by embedding this system in the augmented system discussed below or by establishing a connection between the $\beta$-subsystem for this case with a well-posed interpolation problem. In fact, it is a consequence of our analysis below that there are certain distributions of the auxiliary points $\xi_{i, j}$ that admit nonnegative solutions $\beta_{i, j}$ which give stable discretizations.

The nonsingularity of the full, purely polynomial-based system is very sensitive to the location of the auxiliary points. While there certainly are always distributions of these points that work, it is difficult to pin them down and more difficult 
to determine when they give stable discretizations. If these points are situated sufficiently "upwind," that is, towards the right end of the interval $\left[x_{i}, x_{i+m}\right]$, then everything seems to be all right. The natural way to implement this finite-difference procedure in the transition and outer regions, is to work with the augmented polynomial basis. In this case we get the following.

THEOREM 3.2. For all $h_{m}\left(x_{i}\right)$ sufficiently small and for any $\varepsilon$ positive, the local linear system associated with the $J$-point augmented polynomial-based scheme, exact on $\left\{1, x, \ldots, x^{m+J-2}, \exp \left(-\frac{1}{\varepsilon} \int_{0}^{x} a_{m-1}\right)\right\}$, is nonsingular, and the resulting parameter $\theta_{i}$ satisfies

$$
0 \leq \theta_{i} \leq \frac{1}{\rho_{i}}
$$

Proof. Again it is sufficient to consider the leading-order operator $L_{\varepsilon} u=\varepsilon u^{(m)}+$ $u^{(m-1)}$ and local basis consisting of $P_{m+J-2}$ augmented by $e^{-x / \varepsilon}$. As before, exactness on $P_{m-1}$ implies that $L_{h, \varepsilon}$ must be of the form

$$
L_{h, \varepsilon} u_{i}^{h}=\varepsilon D^{m} u_{i}^{h}+\theta_{i} D^{m-1} u_{i}^{h}+\left(1-\theta_{i}\right) D^{m-1} u_{i+1}^{h} ;
$$

while the requirement on $E(x):=e^{-x / \varepsilon}$ gives

$$
\theta_{i}=\frac{1}{\rho_{i}}\left[1+\frac{D^{m-1} E\left(x_{i+1}\right)}{\varepsilon D^{m} E\left(x_{i}\right)}\right] .
$$

We claim that the following relationship holds for all $\varepsilon>0$ and for any local mesh distribution:

$$
-1<\frac{D^{m-1} E\left(x_{i+1}\right)}{\varepsilon D^{m} E\left(x_{i}\right)}<0 .
$$

That this quantity is negative follows from the fact that

$$
D^{\nu} E\left(x_{j}\right)=E^{(\nu)}(\eta)=(-1)^{\nu} \varepsilon^{-\nu} e^{-\eta / \varepsilon},
$$

for some $\eta$ in $\left(x_{j}, x_{j+\nu}\right)$. To see that this quantity is greater than -1 , define the function

$$
G(\varepsilon):=\frac{D^{m-1} E\left(x_{i+1}\right)}{\varepsilon D^{m} E\left(x_{i}\right)}
$$

It can be verified that $G(0+)=0$ and $G(\varepsilon) \rightarrow-1$, as $\varepsilon \rightarrow \infty$; while, after some manipulations,

$$
G^{\prime}(\varepsilon)=-\frac{D^{m-1} E\left(x_{i+1}\right)}{\varepsilon^{2} D^{m} E\left(x_{i}\right)}>0 .
$$

It follows that $-1<G(\varepsilon)<0$ and $0<\theta_{i}<1 / \rho_{i}$.

The nonsingularity of the subsystem corresponding to the weights, $\beta_{i, j}$, can be established as follows. This system can be written in this case

$$
\begin{aligned}
\sum_{j=1}^{J} \beta_{i, j} & =1, \\
\sum_{j=1}^{J} \beta_{i, j} L_{\varepsilon} \phi\left(\xi_{i, j}\right) & =L_{h, \varepsilon} \phi\left(x_{i}\right), \quad \phi \in\left\{x^{m}, \ldots, x^{m+J-2}\right\} .
\end{aligned}
$$

Suppose that $\left(c_{1}, \ldots, c_{J}\right)$ is a left null vector of the associated matrix. This implies that

$$
L_{\varepsilon} \psi\left(\xi_{i, j}\right)=0, \quad j=1, \ldots, J
$$


where

$$
\psi(x):=c_{1} \frac{x^{m-1}}{(m-1) !}+c_{2} x^{m}+\cdots+c_{J} x^{m+J-2} .
$$

This gives $L_{\varepsilon} \psi=0$, because $L_{\varepsilon} \psi$ is a polynomial of degree at most $J-1$. Thus $\psi$ is in the null space of $L_{\varepsilon}$, which is spanned by $\left\{1, x, \ldots, x^{m-2}, e^{-x / \varepsilon}\right\}$. It then follows that $\psi=0$, from which we get that $c_{1}=\cdots=c_{J}=0$, and the nonsingularity of the matrix is established.

We mention that the $\beta$-subsystem can be analyzed from the standpoint of a weighted quadrature rule, as in [15], with a weight function that is a generalized spline associated with the differential operator $L_{\varepsilon}$. In this connection the positivity of the weights (and higher order of the scheme) for certain choices of auxiliary points, "Gauss points," can be established. We will not go into that here. The schemes for the augmented system are stable regardless; the positivity is of importance only if we deal with the under-determined, pure polynomial system. In summary we would say that while there certainly exist schemes constructed solely using polynomial bases that are stable throughout, it is simpler to use the augmented system in the transition and outer regions.

Truncation error analysis. We sketch some of the details illustrating how the ideas that we have developed can be used to construct and rigorously analyze graded-mesh difference schemes of arbitrarily high uniform rates of convergence, that is, we will prove discretization error bounds of the form

$$
\left\|e^{h}\right\|_{h, \varepsilon} \leq C h^{K}
$$

where $C$ is independent of $\varepsilon$. Fix a positive integer $K$, and construct a graded mesh according to (3.1). We assume that our discretization is constructed, in each of our three subregions, in the following way:

(1) inner region: exact on $P_{m+K}$;

(2) transition region: exact on $P_{m+K-2}$ augmented by the function $\exp \left(-\frac{1}{\varepsilon} \int_{0}^{x} a_{m-1}\right) ;$ and

(3) outer region: exact on $P_{m+K-2}$ and stable.

Note that the inner and transition regions require $(K+1)$-point and $K$-point rules, respectively. In the outer region one can get by with a purely polynomial-based scheme using $(K-1)$ points, if the stability can be assured, or simply use the $K$ point augmented polynomial-based scheme, extending what is done in the transition region. The augmented, layer-type function is only used to insure stability; it is not used in the truncation-error analysis for the outer region.

We assume that we have, in addition to the difference operator $L_{h, \varepsilon}$, discrete auxiliary functionals $\left\{B_{h, \varepsilon, \mu}\right\}_{\mu=1}^{m}$. The details of constructing such functionals can be found, for certain cases, in [5], [15], and [22]. For any such scheme, we define the discretization error, the mesh function $e^{h}:=u-u^{h}$, where $u$ is the true solution of (1.2) and $u^{h}$ is the computed finite-difference approximation to $u$ that solves

$$
L_{h, \varepsilon} u^{h}=\sum_{j=1}^{J} \beta_{i, j} f\left(\xi_{i, j}\right), \quad B_{h, \varepsilon} u^{h}=\gamma^{h} .
$$

It then follows that $e^{h}$ satisfies

$$
L_{h, \varepsilon} e^{h}=\tau^{h}, \quad B_{h, \varepsilon} e^{h}=\sigma^{h},
$$


where

$$
\tau_{i}^{h}:=L_{h, \varepsilon} u\left(x_{i}\right)-\sum_{j=1}^{J} \beta_{i, j} L_{\varepsilon} u\left(\xi_{i, j}\right)
$$

the truncation error, and $\sigma^{h}$ is an appropriately defined truncation term related to the consistency of the discrete auxiliary functionals on $u$.

For the scheme that we have laid out above, we can use our representation result, Theorem 1.4, to establish bounds on the truncation error in the various subregions. We obtain

LEMMA 3.3. There is a constant $C$ such that for $\varepsilon$ sufficiently small, the truncation error defined above satisfies

$$
\left|\tau_{i}^{h}\right| \leq C= \begin{cases}h_{i}^{K+1}+\frac{h_{i}^{K+1}}{\varepsilon^{K+2}} e^{-\frac{1}{\varepsilon} \int_{0}^{x_{i}} a_{m-1}}, & 0 \leq x_{i} \leq x^{*} \\ h_{i}^{K}+e^{-\frac{1}{\varepsilon} \int_{0}^{x_{i}} a_{m-1}}, & x^{*} \leq x_{i} \leq x^{\prime} \\ h^{K}+\frac{h^{K}}{\varepsilon^{K+1}} e^{-\frac{1}{\varepsilon} \int_{0}^{x_{i}} a_{m-1}}, & x^{\prime} \leq x_{i} \leq 1\end{cases}
$$

Proof. For a sufficiently smooth function $\phi$, define the truncation operator $T_{h, \varepsilon}$ by

$$
T_{h, \varepsilon} \phi\left(x_{i}\right):=L_{h, \varepsilon} \phi\left(x_{i}\right)-\sum_{j=1}^{J} \beta_{i, j} L_{\varepsilon} \phi\left(\xi_{i, j}\right) .
$$

Recall from Theorem 1.4 that for all $\varepsilon$ sufficiently small, the solution of (1.2) admits the representation

$$
u=v+\varepsilon^{m-2} w e^{-\frac{1}{\varepsilon} \int_{0}^{x} a_{m-1}}
$$

where $v$ and $w$ have uniformly bounded derivatives. From this it follows, in particular, that

$$
\left|u^{(\nu)}(x)\right| \leq C\left\{1+\varepsilon^{m-\nu-2} e^{-\frac{1}{\varepsilon} \int_{0}^{x} a_{m-1}}\right\} .
$$

In the inner region $\left(0 \leq x_{i} \leq x^{*}\right)$, the discretization is exact on $P_{m+K}$, by assumption; so the leading-order term in the truncation error there will be given by

$$
\begin{aligned}
\left|\tau_{i}^{h}\right| & \approx\left|u^{(m+K+1)}\left(x_{i}\right) T_{h, \varepsilon}\left[\left(x-x_{i}\right)^{m+K+1}\right]\right| \\
& \leq C\left\{1+\varepsilon^{m-2} \frac{1}{\varepsilon^{m+K+1}} e^{-\frac{1}{\varepsilon} \int_{0}^{x_{i}} a_{m-1}}\right\}\left(\varepsilon h_{i}^{K+1}+h_{i}^{K+2}+\cdots\right) \\
& \leq C^{\prime}\left\{\varepsilon h_{i}^{K+1}+\frac{h_{i}^{K+1}}{\varepsilon^{K+2}} e^{-\frac{1}{\varepsilon} \int_{0}^{x_{i}} a_{m-1}}\right\} .
\end{aligned}
$$

In the transition region, the discretization is exact on an augmented polynomial basis, and the truncation error can be bounded:

$$
\left|\tau_{i}^{h}\right| \leq C\left\{T_{h, \varepsilon}\left[\left(x-x_{i}\right)^{m+K-1}\right]+e^{-\frac{1}{\varepsilon} \int_{0}^{x_{i}} a_{m-1}} T_{h, \varepsilon}\left[\varepsilon^{m-2} e^{-\frac{1}{\varepsilon} \int_{x_{i}}^{x} a_{m-1}}\left(x-x_{i}\right)\right]\right\} .
$$

Now in this region, $\varepsilon \leq h_{i}$, and we have

$$
T_{h, \varepsilon}\left[\left(x-x_{i}\right)^{m+K-1}\right] \leq C\left(\varepsilon h_{i}^{K-1}+h_{i}^{K}+\cdots\right) \leq C^{\prime} h_{i}^{K} .
$$


This takes care of the outer part of the solution in this range; the layer part requires a bit more care.

Let $\psi$ denote the local, leading-order term

$$
\psi(x):=\left(x-x_{i}\right) e^{-\frac{1}{\varepsilon} \int_{x_{i}}^{x} a_{m-1}} .
$$

We claim that $T_{h, \varepsilon}\left[\varepsilon^{m-2} \psi\right]=O(1)$. This can be established as follows. Using the representation (2.2) for $L_{h, \varepsilon}$, this expression can be written

$$
T_{h, \varepsilon}\left[\varepsilon^{m-2} \psi\right]=\varepsilon^{m-2}\left\{\varepsilon r_{i} D^{m} \psi\left(x_{i}\right)+\sum_{\nu=0}^{m-1} a_{\nu}^{h}\left(x_{i}\right) D^{\nu} \psi\left(x_{i+1}\right)\right\} .
$$

The terms involving the derivatives through order $(m-1)$ can be bounded using the Mean Value Theorem together with the fact $\rho e^{-\rho} \leq e^{-1}$ for $0<\varepsilon<\infty$. To control the leading term, we note (as in the proof of Theorem 3.2) that

$$
\varepsilon r_{i}=-\frac{D^{m-1} E\left(x_{i+1}\right)}{D^{m} E\left(x_{i}\right)}
$$

where $E(x):=\exp \left(-\frac{1}{\varepsilon} \int_{x_{i}}^{x} a_{m-1}\right)$. Proceeding as in the proof of that theorem, we obtain

$$
\begin{aligned}
\left|\varepsilon^{m-2} \varepsilon r_{i} D^{m} \psi\left(x_{i}\right)\right| & =\left|-\varepsilon^{m-2} D^{m-1} E\left(x_{i+1}\right) \frac{D^{m} \psi\left(x_{i}\right)}{D^{m} E\left(x_{i}\right)}\right| \\
& \leq C \varepsilon^{m-2} \frac{1}{\varepsilon^{m-1}} e^{-\frac{1}{\varepsilon} \int_{x_{i}}^{\varepsilon} a_{m-1}} h_{i}, \quad \text { some } \xi \in\left(x_{i+1}, x_{i+m}\right) \\
& \leq C \frac{h_{i}}{\varepsilon} e^{-\frac{1}{\varepsilon} \int_{x_{i}}^{x_{i+1}} a_{m-1}} \leq C \underline{a}^{-1} e^{-1} .
\end{aligned}
$$

This establishes the bound for the transition region.

In the outer region, we use only the exactness on $P_{m+K-2}$ to obtain

$$
\begin{aligned}
\left|\tau_{i}^{h}\right| & \approx\left|u^{(m+K-1)}\left(x_{i}\right) T_{h, \varepsilon}\left[\left(x-x_{i}\right)^{m+K-1}\right]\right| \\
& \leq C\left\{1+\varepsilon^{m-2} \frac{1}{\varepsilon^{m+K-1}} e^{-\frac{1}{\varepsilon} \int_{0}^{x_{i}} a_{m-1}}\right\}\left(\varepsilon h^{K-1}+h^{K}+\cdots\right) \\
& \leq C\left\{h^{K}+\frac{h^{K}}{\varepsilon^{K+1}} e^{-\frac{1}{\varepsilon} \int_{0}^{x_{i}} a_{m-1}}\right\} .
\end{aligned}
$$

This completes the proof of the last of the desired inequalities.

We note that the estimates obtained in the lemma above are better than what one gets from just a straightforward local Taylor expansion type of analysis. Full use is made of the representation result, that is, we use the fact that $u$ can be decomposed into a uniformly smooth part plus a decaying exponential times another uniformly smooth part, and not just the consequent fact that the derivatives of $u$ can be bounded as in (3.6)

The important consequence for us of the inequalities above is the fact that

$$
\left\|\tau^{h}\right\|_{h, 1}=O\left(h^{K}\right) \text {. }
$$

We establish this as follows. In the inner region, we have

$$
h_{i}=\varepsilon h e^{\frac{1}{K \varepsilon} \int_{0}^{x_{i}} a_{m-1}} .
$$


So

$$
\frac{h_{i}^{K+1}}{\varepsilon^{K+2}} e^{-\frac{1}{\varepsilon} \int_{0}^{x_{i}} a_{m-1}}=h^{K+1} \varepsilon^{-1} e^{\frac{1}{K \varepsilon} \int_{0}^{x_{i}} a_{m-1}},
$$

and

$$
\begin{aligned}
\left\|\tau^{h}\right\|_{\text {inner }, h, 1} & \leq C\left\{h^{K}+h^{K+1} \varepsilon^{-1} \int_{0}^{x^{*}} e^{\frac{1}{K \varepsilon}} \int_{0}^{x} a_{m-1} d x\right\} \\
& \leq C^{\prime}\left\{h^{K}+h^{K+1} e^{\frac{1}{K \varepsilon} \int_{0}^{x^{*}} a_{m-1}}\right\}=(K+1) C^{\prime} h^{K} .
\end{aligned}
$$

Here we have used the definition of $x^{*}$ in (3.2). In a similar way we obtain in the transition region

$$
\begin{aligned}
\left|\tau_{i}^{h}\right| & \leq C\left\{h_{i}^{K}+e^{-\frac{1}{\varepsilon} \int_{0}^{x_{i}} a_{m-1}}\right\} \\
& \leq C\left\{h^{K}+e^{-\frac{1}{\varepsilon} \int_{0}^{x^{*}} a_{m-1}}\right\}=\left(K^{-K}+1\right) C h^{K} .
\end{aligned}
$$

While in the outer region, we have (again using (3.2))

$$
\begin{aligned}
\left\|\tau^{h}\right\|_{\text {outer }, h, 1} & \leq C\left\{h^{K}+\frac{h^{K}}{\varepsilon^{K+1}} \int_{x^{\prime}}^{1} e^{-\frac{1}{\varepsilon} \int_{0}^{x} a_{m-1}} d x\right\} \\
& \leq C^{\prime}\left\{h^{K}+\frac{h^{K}}{\varepsilon^{K}} e^{-\frac{1}{\varepsilon} \int_{0}^{x^{\prime}} a_{m-1}}\right\}=2 C^{\prime} h^{K} .
\end{aligned}
$$

The discrete auxiliary functionals can be handled similarly. The uniform consistency on $N\left(L_{\varepsilon}\right)$ follows, and the strong stability of our discretization is assured. We have established the following.

THEOREM 3.4. Under the assumption that the continuous problem (1.2) is well posed in the sense of (1.3), the discretization (3.5) is strongly uniformly convergent of order $K$ (provided the data of the problem are sufficiently smooth), in the sense that there exist constants $C$ and $h_{0}$ such that the discretization error satisfies

$$
\left\|e^{h}\right\|_{h, \varepsilon} \leq C h^{k}, \quad 0<\varepsilon \leq \varepsilon_{0}, \quad 0<h \leq h_{0} .
$$

The question arises as to whether or not we can get by with one less polynomial element in the inner region, that is, make the scheme exact on $P_{m+K-1}$ there instead of on $P_{m+K}$. It turns out that in that case, the best uniform rate of convergence we can establish is $O\left(h^{K} \ln (1 / h)\right)$, which can be done using simple estimates and the fact that the width of the inner region is $O(\varepsilon \ln (1 / h))$. This rate can be observed numerically for the case of even $K$. When $K$ is odd, however, the smaller space of polynomials is sufficient; there is a local smoothing or cancellation in the leading-order term of the discretization error, as is typical of finite differences.

Finally, we mention that this is just one realization of a family of graded-mesh finite-difference schemes. There are many possible combinations of discretizations and mesh-gradings that can be constructed, and the representation and stability results of Sections 1 and 2 can be used to analyze them.

4. Numerical Results, Conclusions, and Generalizations. Below are reported selected results from numerical experiments that illustrate the performance 
of these schemes. Consider the test problem

$$
\begin{gathered}
\varepsilon u^{\prime \prime}+\frac{2}{2-x} u^{\prime}=\left(\varepsilon-\frac{2}{2-x}\right) e^{1-x}, \\
u(0)=e+2, \quad u(1)=1+2^{-2 / \varepsilon},
\end{gathered}
$$

the solution of which is given by

$$
u(x)=e^{1-x}+(2-x)\left(\frac{2-x}{2}\right)^{2 / \varepsilon} .
$$

The discrete weighted Sobolev norm of the discretization error is listed in Tables $4.1,4.2$, and 4.3 along with the approximate convergence rate

$$
p:=\log _{2} \frac{\left\|\left|e^{h}\right|\right\|_{h, \varepsilon}}{\left\|e^{h / 2} \mid\right\|_{h, \varepsilon}}
$$

for $h=1 / 4,1 / 8, \ldots, 1 / 1024$. The problem (4.1) was discretized using the gradedmesh schemes constructed as in Section 3 with $K=1,2$, and 3. The experiments are reported for the cases $\varepsilon=h^{1 / 2}, \varepsilon=h$, and $\varepsilon=h^{2}$ to cover small and large ratios of $h$ to $\varepsilon$. The computations were done on an IBM $3081 \mathrm{D}$ computer in double-precision arithmetic, which gives around 14 decimal digit accuracy. The predicted $O\left(h^{K}\right)$ uniform accuracy is clearly observed.

TABLE 4.1

Convergence rates for problem (4.1) with $K=1$

\begin{tabular}{c|c|c|c|c|c|c}
\hline & \multicolumn{2}{|c|}{$\varepsilon=h^{1 / 2}$} & \multicolumn{2}{c|}{$\varepsilon=h$} & \multicolumn{2}{c}{$\varepsilon=h^{2}$} \\
\hline$h$ & $\left\|\left.\right|^{h} \mid\right\|_{h, \varepsilon}$ & \multicolumn{1}{c|}{$p$} & $\left\|e^{h}\right\|_{h, \varepsilon}$ & $p$ & $\left\|e^{h}\right\|_{h, \varepsilon}$ & $p$ \\
\hline $1 / 4$ & $.25(-2)$ & .6 & $.92(-2)$ & .1 & $.62(-1)$ & $*$ \\
$1 / 8$ & $.17(-2)$ & .7 & $.88(-2)$ & .7 & $.64(-1)$ & .5 \\
$1 / 16$ & $.10(-2)$ & 1.1 & $.53(-2)$ & .8 & $.45(-1)$ & .9 \\
$1 / 32$ & $.48(-3)$ & 1.3 & $.31(-2)$ & .9 & $.25(-1)$ & 1.0 \\
$1 / 64$ & $.20(-3)$ & 1.3 & $.16(-2)$ & .9 & $.13(-1)$ & 1.0 \\
$1 / 128$ & $.79(-4)$ & 1.4 & $.87(-3)$ & 1.0 & $.66(-2)$ & 1.0 \\
$1 / 256$ & $.30(-4)$ & 1.4 & $.45(-3)$ & 1.0 & $.33(-2)$ & 1.0 \\
$1 / 512$ & $.11(-4)$ & 1.4 & $.23(-3)$ & 1.0 & $.17(-2)$ & 1.0 \\
$1 / 1024$ & $.42(-5)$ & & $.12(-3)$ & & $.84(-3)$ & \\
\hline
\end{tabular}

TABLE 4.2

Convergence rates for problem (4.1) with $K=2$

\begin{tabular}{c|c|c|c|c|c|c}
\hline & \multicolumn{2}{|c|}{$\varepsilon=h^{1 / 2}$} & \multicolumn{2}{c|}{$\varepsilon=h$} & \multicolumn{2}{c}{$\varepsilon=h^{2}$} \\
\hline$h$ & $\left\|e^{h}\right\|_{h, \varepsilon}$ & \multicolumn{1}{c|}{$p$} & $\left\|e^{h}\right\|_{h, \varepsilon}$ & $p$ & $\left\|e^{h}\right\|_{h, \varepsilon}$ & $p$ \\
\hline $1 / 4$ & $.53(-4)$ & .5 & $.16(-2)$ & 1.0 & $.60(-2)$ & .5 \\
$1 / 8$ & $.39(-4)$ & 1.7 & $.80(-3)$ & 1.4 & $.41(-2)$ & 2.2 \\
$1 / 16$ & $.12(-4)$ & 2.3 & $.30(-3)$ & 2.1 & $.88(-3)$ & 2.1 \\
$1 / 32$ & $.24(-5)$ & 2.7 & $.74(-4)$ & 2.1 & $.20(-3)$ & 1.6 \\
$1 / 64$ & $.37(-6)$ & 2.9 & $.17(-4)$ & 2.1 & $.70(-4)$ & 2.2 \\
$1 / 128$ & $.48(-7)$ & 3.0 & $.41(-5)$ & 2.0 & $.14(-4)$ & 2.2 \\
$1 / 256$ & $.61(-8)$ & 3.0 & $.10(-5)$ & 2.0 & $.33(-5)$ & 1.6 \\
$1 / 512$ & $.75(-9)$ & 2.9 & $.26(-6)$ & 2.1 & $.15(-5)$ & 2.2 \\
$1 / 1024$ & $.10(-9)$ & & $.61(-7)$ & & $.23(-6)$ & \\
\hline
\end{tabular}


TABLE 4.3

\begin{tabular}{c|c|c|c|c|c|c}
\multicolumn{6}{c}{ Convergence rates for problem $(4.1)$} & with $K=3$ \\
\hline & \multicolumn{1}{|c|}{$\varepsilon=h^{1 / 2}$} & \multicolumn{2}{c}{$\varepsilon=h$} & \multicolumn{2}{c}{$\varepsilon=h^{2}$} \\
\hline$h$ & $\left\|e^{h}\right\|_{h, \varepsilon}$ & $p$ & $\left\|e^{h}\right\|_{h, \varepsilon}$ & $p$ & $\left\|e^{h}\right\|_{h, \varepsilon}$ & $p$ \\
\hline $1 / 4$ & $.58(-7)$ & $*$ & $.88(-5)$ & 1.2 & $.40(-4)$ & 2.6 \\
$1 / 8$ & $.97(-7)$ & 1.2 & $.38(-5)$ & 2.5 & $.67(-5)$ & 3.6 \\
$1 / 16$ & $.42(-7)$ & 2.2 & $.68(-6)$ & 3.1 & $.54(-6)$ & 2.6 \\
$1 / 32$ & $.92(-8)$ & 2.9 & $.77(-7)$ & 3.1 & $.88(-7)$ & 3.3 \\
$1 / 64$ & $.12(-8)$ & 3.3 & $.93(-8)$ & 3.1 & $.92(-8)$ & 2.5 \\
$1 / 128$ & $.12(-9)$ & 2.9 & $.11(-8)$ & 3.1 & $.16(-8)$ & 3.3 \\
$1 / 256$ & $.02(-9)$ & & $.14(-9)$ & & $.17(-9)$ & \\
\hline
\end{tabular}

We would cite two main conclusions of our work. First, while we do not have as nice a stability theory for discretizations of singularly perturbed differential equations as we do for nonsingularly perturbed equations (where consistency plus compactness imply stability), we have shown that we do have strong uniform stability of these schemes over a much wider range than previously realized. Second, it is possible to construct (and rigorously analyze) polynomial-based graded-mesh finite-difference schemes of arbitrarily high order of accuracy, uniform in $\varepsilon$.

We have not analyzed the most general situation to which these ideas can be applied. Our results can be generalized in at least three ways. First, nonlinear differential equations of the form

$$
\varepsilon u^{(m)}+a_{m-1} u^{(m-1)}=f\left(x, u, \ldots, u^{(m-2)}\right)
$$

can be shown to possess the same types of stability properties as the operators considered here provided their linearizations around an isolated solution are Lipschitz continuous. The technique is due to Keller [12].

Second, the entire analysis can be carried out with respect to weaker norms. Suppose that $\||\cdot|\|_{\varepsilon}^{\prime}$ is any norm that satisfies (for sufficiently smooth $u$ )

$$
\|u\|\left\|_{\varepsilon}^{\prime} \leq C\right\|\|u\| \|_{\varepsilon}
$$

We can establish continuous and discrete stability results involving such a norm which are analogous to those of Theorems 1.2 and 2.4. We also get a representation result of the form

$$
u=v+\varepsilon^{L} w e^{-\frac{1}{\varepsilon} \int_{0}^{x} a_{m-1},}
$$

where $L$ is a nonnegative integer that depends on the nature of the stability and auxiliary conditions.

Consider for example the model problem

$$
L_{\varepsilon} u=\varepsilon u^{(4)}+u^{(3)}, \quad B_{\varepsilon} u=\left(u(0), u^{\prime}(0), u(1), u^{\prime}(1)\right) .
$$

It is shown in [17] that this pair satisfies a strong uniform stability property of the form

$$
\|\| u \|_{\varepsilon}^{\prime} \leq C\left\{\left\|L_{\varepsilon} u\right\|_{1}+\left|B_{\varepsilon} u\right|\right\}
$$

for

$$
\|u\|_{\varepsilon}^{\prime}=\|u\|_{\infty} \quad \text { or } \quad\|u\|_{\varepsilon}^{\prime}=\max \left\{\|u\|_{\infty},\left\|u^{\prime}\right\|_{\infty}\right\}
$$


but not for

$$
\||u|\|_{\varepsilon}^{\prime}=\max \left\{\|u\|_{\infty},\left\|u^{\prime}\right\|_{\infty},\left\|u^{\prime \prime}\right\|_{\infty}\right\}
$$

or

$$
\|\| u\|\|_{\varepsilon}^{\prime}=\max \left\{\|u\|_{\infty},\left\|u^{\prime}\right\|_{\infty},\left\|u^{\prime \prime}\right\|_{\infty}, \varepsilon\left\|u^{\prime \prime \prime}\right\|_{\infty}\right\} .
$$

The representation result for the associated boundary value problem takes the form

$$
u=v+\varepsilon w e^{-x / \varepsilon} .
$$

The same differential operator with the multipoint auxiliary conditions

$$
B_{\varepsilon} u=(u(0), u(1 / 3), u(2 / 3), u(1))
$$

will be stable with respect to the $L^{\infty}$-norm but not with respect to any norms involving higher derivatives. And for this problem, the solutions are represented by

$$
u=v+w e^{-x / \varepsilon} \text {. }
$$

A third possible generalization would be to consider singularly perturbed differential operators of the form

$$
L_{\varepsilon} u=\varepsilon M u+N u,
$$

where $M$ and $N$ are linear and of exact orders $m$ and $n(<m)$ with nonvanishing leading coefficients. One can obtain analogous stability results with respect to appropriate norms. The representation result involves several layer functions, the exponent functions of which are related to a certain characteristic equation (cf. [18] or [23]).

Department of Mathematical Sciences

Kent State University

Kent, Ohio 44242

E-mail: gartland@kent.edu

1. U. ASCHER \& R. WEISS, "Collocation for singular perturbation problems I: First order systems with constant coefficients," SIAM J. Numer. Anal., v. 20, 1983, pp. 537-557.

2. U. ASCHER \& R. WEISS, "Collocation for singular perturbation problems II: Linear first order systems without turning points," Math. Comp., v. 43, 1984, pp. 157-187.

3. U. ASCHER \& R. WEISS, "Collocation for singular perturbation problems III: Nonlinear problems without turning points," SIAM J. Sci. Statist. Comput., v. 5, 1984, pp. 811-829.

4. A. E. Berger, J. M. SOLOMON \& M. CiMENT, "Uniformly accurate difference methods for a singular perturbation problem," in Boundary and Interior Layers Computational and Asymptotic Methods, Proc. BAIL I Conf. (J. J. H. Miller, ed.), Boole Press, Dublin, 1980.

5. E. J. DOEDEL, "The construction of finite difference approximations to ordinary differential equations," SIAM J. Numer. Anal., v. 15, 1978, pp. 450-465.

6. H. ESSER, "Stabilitätsungleichungen für Diskretisierungen von Randwertaufgaben gewöhnlicher Differentialgleichungen," Numer. Math., v. 28, 1977, pp. 69-100.

7. L. S. Frank, "Difference singular perturbations-I. A priori estimates," J. Math. Anal. Appl., v. 70,1979 , pp. $180-235$.

8. E. C. Gartland, JR., Strong Stability and a Rrepresentation Result for a Singular Perturbation Problem, Technical Report AMS 87-1, January, 1987, Department of Mathematics, Southern Methodist University.

9. E. C. GARTLAND, JR., "Uniform high-order difference schemes for a singularly perturbed two-point boundary value problem," Math. Comp., v. 48, 1987, pp. 551 564 .

10. E. C. Gartland, JR., "Strong stability of compact discrete boundary value problems via exact discretizations," SIAM J. Numer. Anal., v. 25, 1988, pp. 111-123. 
11. R. D. GRIGORIEFF, "Über die Koerzitivität gewöhnlicher Differenzenoperatoren und die Konvergenz von Mehrschrittverfahren," Numer. Math., v. 15, 1970, pp. 196-218.

12. H. B. KELLER, "Approximation methods for nonlinear problems with application to twopoint boundary value problems," Math. Comp., v. 29, 1975, pp. 464-474.

13. H.-O. KREISS, "Difference approximations for boundary and eigenvalue problems for ordinary differential equations," Math. Comp., v. 26, 1972, pp. 605-624.

14. H.-O. KREISS, N. K. NICHOLS \& D. L. BROWN, "Numerical methods for stiff two-point boundary value problems," SIAM J. Numer. Anal., v. 23, 1986, pp. 325-368.

15. R. E. LYNCH \& J. R. RICE, "A high-order difference method for differential equations," Math. Comp., v. 34, 1980, pp. 333-372.

16. P. A. MARKowich \& C. A. Ringhofer, "Collocation methods for boundary value problems on 'long' intervals," Math. Comp., v. 40, 1983, pp. 123-150.

17. K. NiEDERDRENK \& H. YSERENTANT, "Die gleichmässige Stabilität singulär gestörter diskreter und kontinuierlicher Randwertprobleme," Numer. Math., v. 41, 1983, pp. 223-253.

18. R. E. O'MALley, JR., Introduction to Singular Perturbations, Academic Press, New York, 1974.

19. M. R. OSBORNE, "Minimizing truncation error in finite difference approximations to ordinary differential equations," Math. Comp., v. 21, 1967, pp. 133-145.

20. A. H. SChatz \& L. B WAHLBin, "Maximum norm estimates in the finite element method on plane polygonal domains. Part 2, Refinements," Math. Comp., v. 33, 1979, pp. 465-492.

21. D. R. SMith, Singular Perturbation Theory: An Introduction with Applications, Cambridge Univ. Press, Cambridge, 1985.

22. B. SWARTZ, "Compact, implicit difference schemes for a differential equation's side conditions," Math. Comp., v. 35, 1980, pp. 733-746.

23. H. L. TURRITIN, "Asymptotic solutions of certain ordinary differential equations associated with multiple roots of the characteristic equation," Amer. J. Math., v. 58, 1936, pp. 364-378.

24. R. VULANOVIĆ, "On numerical solution of a type of singularly perturbed boundary value problem by using a special discretization mesh," Univ. u Novom Sadu Zb. Rad. Prirod.-Mat. Fak. Ser. Mat., v. 13, 1983, pp. 187-201. 